\title{
Modeling topic control to detect influence in conversations using nonparametric topic models
}

\author{
Viet-An Nguyen • Jordan Boyd-Graber • Philip Resnik • \\ Deborah A. Cai · Jennifer E. Midberry • Yuanxin Wang
}

Received: 17 August 2012 / Accepted: 5 September 2013 / Published online: 23 October 2013

(C) The Author(s) 2013

\begin{abstract}
Identifying influential speakers in multi-party conversations has been the focus of research in communication, sociology, and psychology for decades. It has been long acknowledged qualitatively that controlling the topic of a conversation is a sign of influence. To capture who introduces new topics in conversations, we introduce SITS - Speaker Identity for Topic Segmentation-a nonparametric hierarchical Bayesian model that is capable of discovering (1) the topics used in a set of conversations, (2) how these topics are shared across conversations, (3) when these topics change during conversations, and (4) a speakerspecific measure of "topic control". We validate the model via evaluations using multiple datasets, including work meetings, online discussions, and political debates. Experimental results confirm the effectiveness of SITS in both intrinsic and extrinsic evaluations.
\end{abstract}

Editors: Winter Mason, Jennifer Wortman Vaughan, and Hanna Wallach.

V.-A. Nguyen ( $\varangle)$

Department of Computer Science, University of Maryland, College Park, MD, USA

e-mail: vietan@cs.umd.edu

J. Boyd-Graber

iSchool and UMIACS, University of Maryland, College Park, MD, USA

e-mail: jbg@umiacs.umd.edu

P. Resnik

Department of Linguistics and UMIACS, University of Maryland, College Park, MD, USA

e-mail: resnik@umd.edu

D.A. Cai · J.E. Midberry · Y. Wang

School of Media and Communication, Temple University, Philadelphia, PA, USA

D.A. Cai

e-mail: debcai@temple.edu

J.E. Midberry

e-mail: jmidberry@gmail.com

Y. Wang

e-mail: yuanxin@gmail.com 
Keywords Bayesian nonparametrics · Influencer detection · Topic modeling · Topic segmentation · Gibbs sampling

\section{Influencing conversations by controlling the topic}

Conversation, interactive discussion between two or more people, is one of the most essential and common forms of communication in our daily lives. ${ }^{1}$ One of the many functions of conversations is influence: having an effect on the belief, opinions or intentions of other conversational participants. Using multi-party conversations to study and identify influencers, the people who influence others, has been the focus of researchers in communication, sociology, and psychology (Katz and Lazarsfeld 1955; Brooke and Ng 1986; Weimann 1994), who have long acknowledged that there is a correlation between the conversational behaviors of a participant and how influential he or she is perceived to be by others (Reid and Ng 2000).

In an early study on this topic, Bales (1970) argues "To take up time speaking in a small group is to exercise power over the other members for at least the duration of the time taken, regardless of the content." This statement asserts that structural patterns such as speaking time and activeness of participation are good indicators of power and influence in a conversation. Participants who talk most during a conversation are often perceived as having more influence (Sorrentino and Boutiller 1972; Regula and Julian 1973; Daley et al. 1977; Ng et al. 1993), more leadership ability (Stang 1973; Sorrentino and Boutiller 1972), more dominance (Palmer 1989; Mast 2002) and more control of the conversation (Palmer 1989). Recent work using computational methods also confirms that structural features such as number of turns and turn length are among the most discriminative features to classify whether a participant is influential or not (Rienks et al. 2006; Biran et al. 2012).

However, it is wrong to take Bales's claim too far; the person who speaks loudest and longest is not always the most powerful. In addition to structural patterns, the characteristics of language used also play an important role in establishing influence and controlling the conversation ( $\mathrm{Ng}$ and Bradac 1993). For example, particular linguistic choices such as message clarity, powerful and powerless language (Burrel and Koper 1998), and language intensity (Hamilton and Hunter 1998) in a message can increase influence. More recently, Huffaker (2010) showed that linguistic diversity expressed by lexical complexity and vocabulary richness has a strong relationship with leadership in online communities. To build a classifier to detect influencers in written online conversations, Biran et al. (2012) also propose to use a set of content-based features to capture various participants' conversational behaviors, including persuasion and agreement/disagreement.

Among many studied behaviors, topic control and management is considered one of the most effective ways to control the conversation (Planalp and Tracy 1980). Palmer (1989) shows that the less related a participants' utterances are to the immediate topic, the more dominant they are, and then argues, "the ability to change topical focus, especially given strong cultural and social pressure to be relevant, means having enough interpersonal power to take charge of the agenda." Recent work by Rienks et al. (2006) also shows that topic change, among other structural patterns discussed above, is the most robust feature in detecting influencers in small group meetings.

In this article, we introduce a new computational model capturing the role of topic control in participants' influence of conversations. Speaker Identity for Topic Segmentation

\footnotetext{
${ }^{1}$ This paper significantly revises and extends the work described in Nguyen et al. (2012).
} 
(SITS), a hierarchical Bayesian nonparametric model, uses an unsupervised statistical approach which requires few resources and can be used in many domains without extensive training and annotation. More important, SITS incorporates an explicit model of speaker behavior by characterizing quantitatively individuals' tendency to exercise control over the topic of conversation (Sect. 3). By focusing on topic changes in conversations, we go beyond previous work on influencers in two ways:

- First, while structural statistics such as number of turns, turn length, speaking time etc are relatively easy to extract from a conversation, defining and detecting topic changes is less well understood. Topic, by itself, is a complex concept (Blei et al. 2003; Kellermann 2004). In addition, despite the large number of techniques proposed trying to divide a document into smaller, topically coherent segments (Purver 2011), topic segmentation is still an open research problem. Most previous computational methods for topic discovery and topic segmentation focus on content, ignoring the speaker identities. We show that we can capture conversational phenomena and influence better by explicitly modeling behaviors of participants.

- Second, the conversation is often controlled explicitly, to some extent, by a subset of participants. For example, in political debates questions come from the moderator(s), and candidates typically have a fixed time to respond. These imposed aspects of conversational structure decrease the value of more easily extracted structural statistics for a variety of conversation types; observe, for example, that similar properties of the conversation can also be observed when looking at hosts and guests in televised political discussion shows such as CNN's Crossfire.

Applying SITS on real-world conversations (Sect. 4), we show that this modeling approach is not only more effective than previous methods on traditional topic segmentation (Sect. 5), but also more intuitive in that it is able to capture an important behavior of individual speakers during conversations (Sect. 6). We then show that using SITS to model topic control improves influencer detection (Sect. 7). Taking quantitative and qualitative analysis together, the pattern of results suggests that our approach holds significant promise for further development; we discuss directions for future work in Sect. 8.

\section{What is an influencer?}

\subsection{Influencer definition}

In most research on persuasion and power, an influencer attempts to gain compliance from others or uses tactics to shape the opinions, attitudes, or behaviors of others (Scheer and Stern 1992; Schlenker et al. 1976). In research on social media, such as blogs and Twitter, measurements such as the number of followers or readers serve as a proxy for influence (Alarcon-del Amo et al. 2011; Booth and Matic 2011; Trammell and Keshelashvili 2005). Others have studied what influencers say; Drake and Moberg (1986) demonstrated that linguistic influence differs from attempts to influence that rely on power and exchange relationships. In interactions with targets, influencers may rely more on linguistic frames and language than on resources offered, which is proposed as the requirement for influence by exchange theorists (Blau 1964; Foa and Foa 1972; Emerson 1981).

We define an influencer as someone who has persuasive ability over where an interaction is headed, what topics are covered, and what positions are espoused within that interaction. In the same way that persuasion shapes, reinforces, or changes attitudes or beliefs, an influencer shapes, reinforces, or changes the direction of the interaction. An influencer within 
an interaction is someone who may introduce new ideas or arguments into the conversation that others pick up on and discuss (shapes new directions through topic shift), may express arguments about an existing topic that others agree to and further in the discussion (i.e., reinforces the direction), or may provide counter-arguments that others agree to and perpetuate, thereby redirecting where the topic of conversation is headed (i.e., changes the direction of the conversation).

\subsection{Data scope and characteristics}

We are interested in influence in turn-taking, multiparty discussions. This is a broad category including political debates, business meetings, online chats, discussions, conference panels, and many TV or radio talk shows. More formally, such datasets contain $C$ conversations. A conversation $c$ has $T_{c}$ turns, each of which is a maximal uninterrupted utterance by one speaker. ${ }^{2}$ In each turn $t \in\left[1, T_{c}\right]$, a speaker $a_{c, t}$ utters $N_{c, t}$ words $\boldsymbol{w}_{c, t}=\left\{w_{c, t, n} \mid n \in\right.$ $\left.\left[1, N_{c, t}\right]\right\}$. Each word is from a vocabulary of size $V$, and there are $M$ distinct speakers.

\section{Modeling topic shift}

In this section, we describe SITS, a hierarchical nonparametric Bayesian model for topic segmentation that takes into consideration speaker identities, allowing us to characterize speakers' topic control behavior over the course of the discussion (Nguyen et al. 2012). We begin with an overview of the topic segmentation problem and some related work. We then highlight the differences between SITS and previous approaches and describe the generative process and the inference technique we use to estimate the model.

\subsection{Topic segmentation and modeling approaches}

Whether in an informal situation or in more formal settings such as a political debate of business meeting, a conversation is often not about just one thing: topics evolve and are replaced as the conversation unfolds. Discovering this hidden structure in conversations is a key problem for building conversational assistants (Tur et al. 2010) and developing tools that summarize (Murray et al. 2005) and display (Ehlen et al. 2007) conversational data. Understanding when and how the topics change also helps us study human conversational behaviors such as individuals' agendas (Boydstun et al. 2013), patterns of agreement and disagreement (Hawes et al. 2009; Abbott et al. 2011), relationships among conversational participants (Ireland et al. 2011), and dominance and influence among participants (Palmer 1989; Rienks et al. 2006).

One of the most natural ways to capture conversational structure is topic segmentationthe task of "automatically dividing single long recordings or transcripts into shorter, topically coherent segments" (Purver 2011). There are broadly two basic approaches previous work has used to tackle this problem. The first approach focuses on identifying discourse markers which distinguish topical boundaries in the conversations. There are certain cue phrases such as well, now, that reminds me, etc. that explicitly indicate the end of one topic or the beginning of another (Hirschberg and Litman 1993; Passonneau and Litman 1997). These markers can also serve as features for a discriminative classifier (Galley et al. 2003) or observed variables in generative model (Dowman

\footnotetext{
${ }^{2}$ Note the distinction from phonetic utterances, which by definition are bounded by silence.
} 
et al. 2008). However, in practice the discourse markers that are most indicative of topic change often depend heavily on the domain of the data (Purver 2011). This drawback makes methods solely relying on these markers difficult to adapt to new domains or settings.

Our method follows the second general approach, which relies on the insight that topical segments evince lexical cohesion (Halliday and Hasan 1976). Intuitively, words within a segment will look more like their neighbors than like words in other segments. This has been a key idea in previous work. Morris and Hirst (1991) try to determine the structure of text by finding "lexical chains" which consists of units of text that are about the same thing. The often used text segmentation algorithm TextTiling (Hearst 1997) exploits this insight to compute the lexical similarity between adjacent sentences. More recent improvements to this approach include using different lexical similarity metrics like LSA (Choi et al. 2001; Olney and Cai 2005) and improving feature extraction for supervised methods (Hsueh et al. 2006). It also inspires unsupervised models using bags of words (Purver et al. 2006), language models (Eisenstein and Barzilay 2008), and shared structure across documents (Chen et al. 2009).

We also use lexical cohesion using a probabilistic topic modeling method (Blei et al. 2003; Blei 2012). The approach we take is unsupervised, so it requires few resources and is applicable in many domains without extensive training. Following the literature on topic modeling, we define each topic as a multinomial distribution over the vocabulary. Like previous generative models proposed for topic segmentation (Purver et al. 2006), each turn is considered a bag of words generated from an admixture of topics and topics are shared across different turns within a conversation or across different conversations. ${ }^{3}$ In addition, we take a Bayesian nonparametric approach (Müller and Quintana 2004) to allow the number of topics to be unbounded, in order to better represent the observed data.

The settings described above are still consistent with those in popular topic models such as latent Dirichlet allocation (Blei et al. 2003, LDA) or hierarchical Dirichlet processes (Teh et al. 2006, HDP), in which turns in a conversation are considered independent. In practice, however, this is not the case. Obviously the topics of a turn at time $t$ are highly correlated with those of the turn at $t+1$. To address this issue, there have been several recent attempts trying to capture the temporal dynamics within a document. Du et al. (2010) propose Sequential LDA to study how topics within a document evolve over its structure. It uses the nested two-parameter Poisson Dirichlet process (PDP) to model the progressive dependency between consecutive part of a document, which can capture the continuity of topical flow in a document nicely but does not capture the topic change explicitly. Fox et al. (2008) proposed Sticky HDP-HMM, which is an extension of HDP-HMM (Teh et al. 2006) for the problem of speaker diarization involving segmenting an audio recording into intervals associated with individual speakers. Applying to the conversational setting, Sticky HDP-HMM associates each turn with a single topic; this is a strong assumption since people tend to talk about more than one thing in a turn, especially in political debates. We will, however, use it as one of the baselines in our topic segmentation experiment (Sect. 5). A related problem is to discover how topics themselves change over time (Blei and Lafferty 2006; Wang et al. 2008; Ren et al. 2008; Ahmed and Xing 2008, 2010), e.g., documents that talk about "physics" in 1900 will use very different terms than "physics" in 2000. These models assume documents are much longer and that topics evolve much more slowly than in a conversation.

\footnotetext{
${ }^{3}$ The "bag of words" treatment of linguistic utterances is widely used, but of course a gross simplification. In other research, we have investigated nonparametric models capturing arbitrary-length phrases (Hardisty et al. 2010) and syntactic topic models (Boyd-Graber and Blei 2008); integrating linguistically richer models with SITS is a topic for future work.
} 


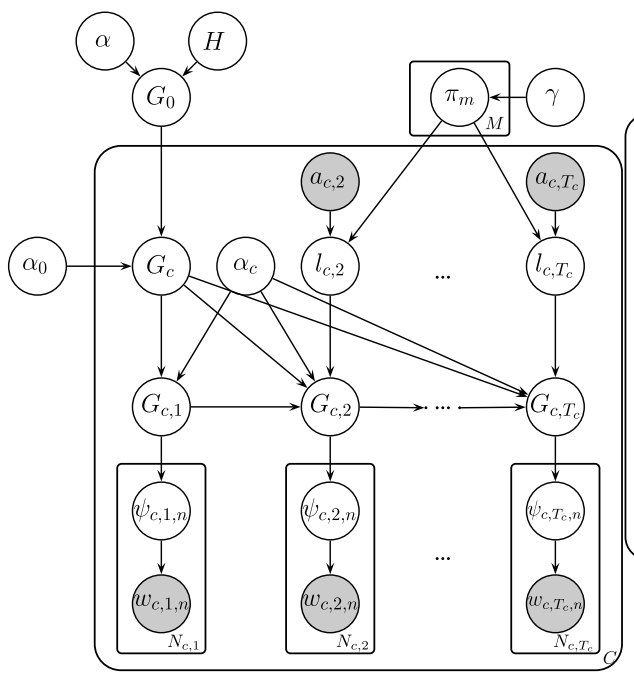

(a)

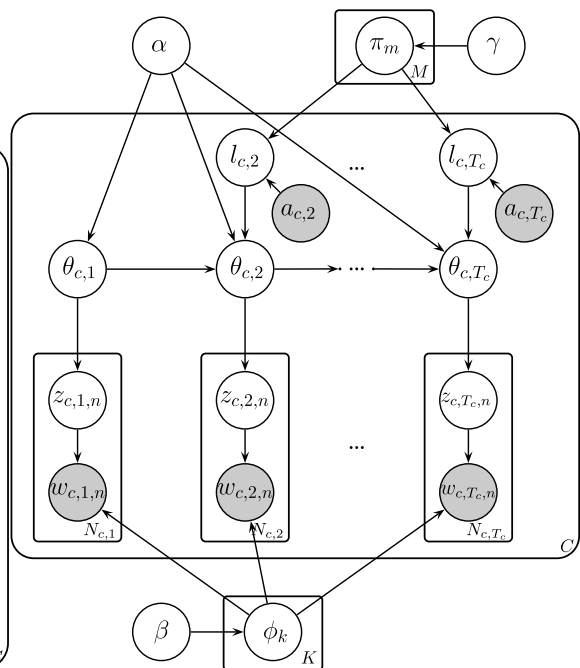

(b)

Fig. 1 Plate diagrams of our proposed models: (a) nonparametric SITS; (b) parametric SITS. Nodes represent random variables (shaded nodes are observed); lines are probabilistic dependencies. Plates represent repetition. The innermost plates are turns, grouped in conversations

Moreover, many of these methods do not explicitly model the changes of the topics within a document or conversation. To address this, we endow each turn with a binary latent variable $l_{c, t}$, called the topic shift indicator (Purver et al. 2006). This latent variable signifies whether in this turn the speaker changed the topic of the conversation. In addition, to capture the topic-controlling behavior of the speakers across different conversations, we further associate each speaker $m$ with a latent topic shift tendency denoted by $\pi_{m}$. Informally, this variable is intended to capture the propensity of a speaker to effect a topic shift. Formally, it represents the probability that the speaker $m$ will change the topic (distribution) of a conversation. In the remainder of this section, we will describe the model in more detail together with the inference techniques we use.

\subsection{Generative process of SITS}

SITS is a generative model of multiparty discourse that jointly discovers topics and speakerspecific topic shifts from an unannotated corpus (Fig. 1a). As in the hierarchical Dirichlet process (Teh et al. 2006), we allow an unbounded number of topics to be shared among the turns of the corpus. Topics are drawn from a base distribution $H$ over multinomial distributions over the vocabulary of size $V ; H$ is a finite Dirichlet distribution with symmetric prior $\lambda$. Unlike the HDP, where every document (here, every turn) independently draws a new multinomial distribution from a Dirichlet process, the social and temporal dynamics of a conversation, as specified by the binary topic shift indicator $l_{c, t}$, determine when new draws happen.

Generative process The formal generative process is:

1. For speaker $m \in[1, M]$, draw speaker topic shift probability $\pi_{m} \sim \operatorname{Beta}(\gamma)$

2. Draw the global topic distribution $G_{0} \sim \operatorname{DP}(\alpha, H)$ 
3. For each conversation $c \in[1, C]$

(a) Draw a conversation-specific topic distribution $G_{c} \sim \operatorname{DP}\left(\alpha_{0}, G_{0}\right)$

(b) For each turn $t \in\left[1, T_{c}\right]$ with speaker $a_{c, t}$

i. If $t=1$, set the topic shift indicator $l_{c, t}=1$. Otherwise, draw $l_{c, t} \sim$ Bernoulli $\left(\pi_{a_{c, t}}\right)$.

ii. If $l_{c, t}=1$, draw $G_{c, t} \sim \operatorname{DP}\left(\alpha_{c}, G_{c}\right)$. Otherwise, set $G_{c, t} \equiv G_{c, t-1}$.

iii. For each word index $n \in\left[1, N_{c, t}\right]$

- Draw a topic $\psi_{c, t, n} \sim G_{c, t}$

- Draw a token $w_{c, t, n} \sim \operatorname{Multinomial}\left(\psi_{c, t, n}\right)$.

The hierarchy of Dirichlet processes allows statistical strength to be shared across contexts; within a conversation and across conversations. The per-speaker topic shift tendency $\pi_{m}$ allows speaker identity to influence the evolution of topics.

Intuitively, SITS generates a conversation as follows: At the beginning of a conversation $c$, the first speaker $a_{c, 1}$ draws a distribution over topics $G_{c, 1}$ from the base distribution, and uses that topic distribution to draw a topic $\psi_{c, 1, n}$ for each token $w_{c, 1, n}$. Subsequently, at turn $t$, speaker $a_{c, t}$ will first flip a speaker-specific biased coin $\pi_{a_{c, t}}$ to decide whether $a_{c, t}$ will change the topic of the conversation. If the coin comes up tails $\left(l_{c, t}=0\right), a_{c, t}$ will not change the conversation topic and uses the previous turn's topic distribution $G_{c, t-1}$ to generate turn $t$ 's tokens. If, on the other hand, the coin comes up heads $\left(l_{c, t}=1\right), a_{c, t}$ will change the topic by drawing a new topic distribution $G_{c, t}$ from the conversation-specific collection of topics $\operatorname{DP}\left(\alpha_{c}, G_{c}\right)$.

Segmentation notation To make notation more concrete and to connect our model with topic segmentation, we introduce the notion of segments in a conversation. A segment $s$ of conversation $c$ is a sequence of turns $\left[\tau, \tau^{\prime}\right]$ such that

$$
\left\{\begin{array}{l}
l_{c, \tau}=l_{c, \tau^{\prime}+1}=1 \\
l_{c, t}=0, \quad \forall t \in\left[\tau+1, \tau^{\prime}\right]
\end{array}\right.
$$

When $l_{c, t}=0, G_{c, t}$ is the same as $G_{c, t-1}$ and all topics (i.e. multinomial distributions over words) $\left\{\psi_{c, t, n} \mid n \in\left[1, N_{c, t}\right]\right\}$ that generate words in turn $t$ and the topics $\left\{\psi_{c, t-1, n^{\prime}} \mid n^{\prime} \in\right.$ $\left.\left[1, N_{c, t-1}\right]\right\}$ that generate words in turn $t-1$ come from the same distribution. Thus, all topics used in a segment $s$ are drawn from a single segment-specific probability measure $G_{c, s}$,

$$
G_{c, s} \mid l_{c, 1}, l_{c, 2}, \ldots, l_{c, T_{c}}, \alpha_{c}, G_{c} \sim \operatorname{DP}\left(\alpha_{c}, G_{c}\right)
$$

A visual illustration of these notations can be found in Fig. 2. For notational convenience, $S_{c}$ denotes the number of segments in conversation $c$, and $s_{t}$ denotes the segment index of turn $t$. We emphasize that all segment-related notations are derived from the posterior over the topic shifts $l$ and not part of the model itself.

\subsection{Inference for SITS}

To find the latent variables that best explain observed data, we use Gibbs sampling, a widely used Markov chain Monte Carlo inference technique (Neal 2000; Resnik and Hardisty 2010). The state space in our Gibbs sampler consists of the latent variables for topic indices assigned to all tokens $z=\left\{z_{c, t, n}\right\}$ and topic shifts assigned to turns $\boldsymbol{l}=\left\{l_{c, t}\right\}$. We marginalize over all other latent variables. For each iteration of the sampling process, we loop over each turn in each conversation. For a given turn $t$ in conversation $c$, we first sample the topic 


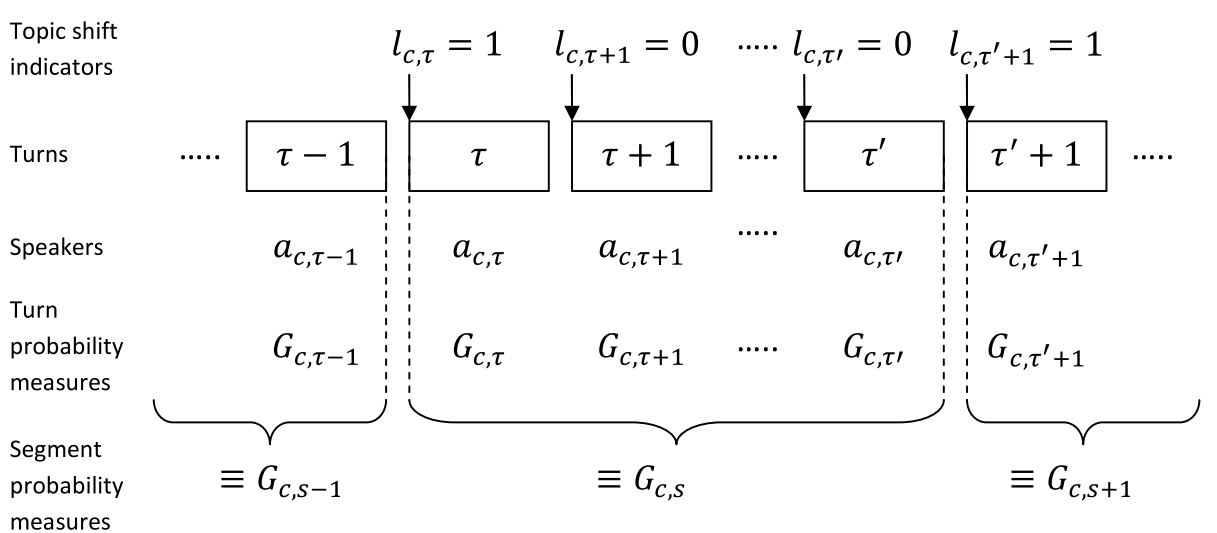

Fig. 2 Diagram of notation for topic shift indicators and conversation segments: Each turn is associated with a latent binary variable topic shift indicator $l$ specifying whether the topic of the turn is shifted. In this example, topic shifts occur in turns $\tau$ and $\tau^{\prime}+1$. As a result, the topic shift indicators of turn $\tau$ and $\tau^{\prime}+1$ are equal to 1 (i.e. $l_{c, \tau}=l_{c, \tau^{\prime}+1}=1$ ) and the topic shift indicators of all turns in between are 0 (i.e. $l_{c, t}=0, \forall t \in\left[\tau+1, \tau^{\prime}\right]$ ). Turns $\left[\tau, \tau^{\prime}\right]$ form a segment $s$ in which all topic distributions $G_{c, \tau}, G_{c, \tau+1}, \ldots, G_{c, \tau^{\prime}}$ are the same and are denoted collectively as $G_{c, s}$

shift indicator variable $l_{c, t}$ (Sect. 3.3.2) and then sample the topic assignment $z_{c, t, n}$ for each token in the turn (Sect. 3.3.1). Here, we only present the conditional sampling equations; for details on how these are derived, see the Appendix A.

\subsubsection{Sampling topic assignments}

In Bayesian nonparametrics, the Chinese restaurant process (CRP) metaphor is often used to explain the clustering effect of the Dirichlet process (Ferguson 1973). The CRP is an exchangeable distribution over partitions of integers, which facilitates Gibbs sampling (Neal 2000) (as we will see in (2)). When used in topic models, each Chinese restaurant consists of infinite number of tables, each of which corresponds to a topic. Customers, each of which corresponds to a token, are assigned to tables and if two tokens are assigned to the same table: they share the same topic.

The CRP has a "rich get richer" property, which means that tables with many customers will attract yet more customers - a new customer will sit at an existing table with probability proportional to the number of customers currently at the table. The CRP has no limit on the number of tables; when a customer needs to be seated, there is always a probabilityproportional to the Dirichlet parameter $\alpha$ - that it will be seated at a new table. When a new table is formed, it is assigned a "dish"; this is a draw from the Dirichlet process's base distribution. In a topic model, this atom associated with a new table is a multinomial distribution over word types. In a standard, non-hierarchical CRP, this multinomial distribution comes from a Dirichlet distribution.

But it doesn't have to-hierarchical nonparametric models extend the metaphor further by introducing a hierarchy of restaurants (Teh et al. 2006; Teh 2006), where the base distribution of one restaurant can be another restaurant. This is where things can get tricky. Instead of having a seating assignment, a customer now has a seating path and is potentially responsible for spawning new tables in every restaurant. In SITS there are restaurants for the current segment, the conversation, and the entire corpus, as shown in Fig. 3. 


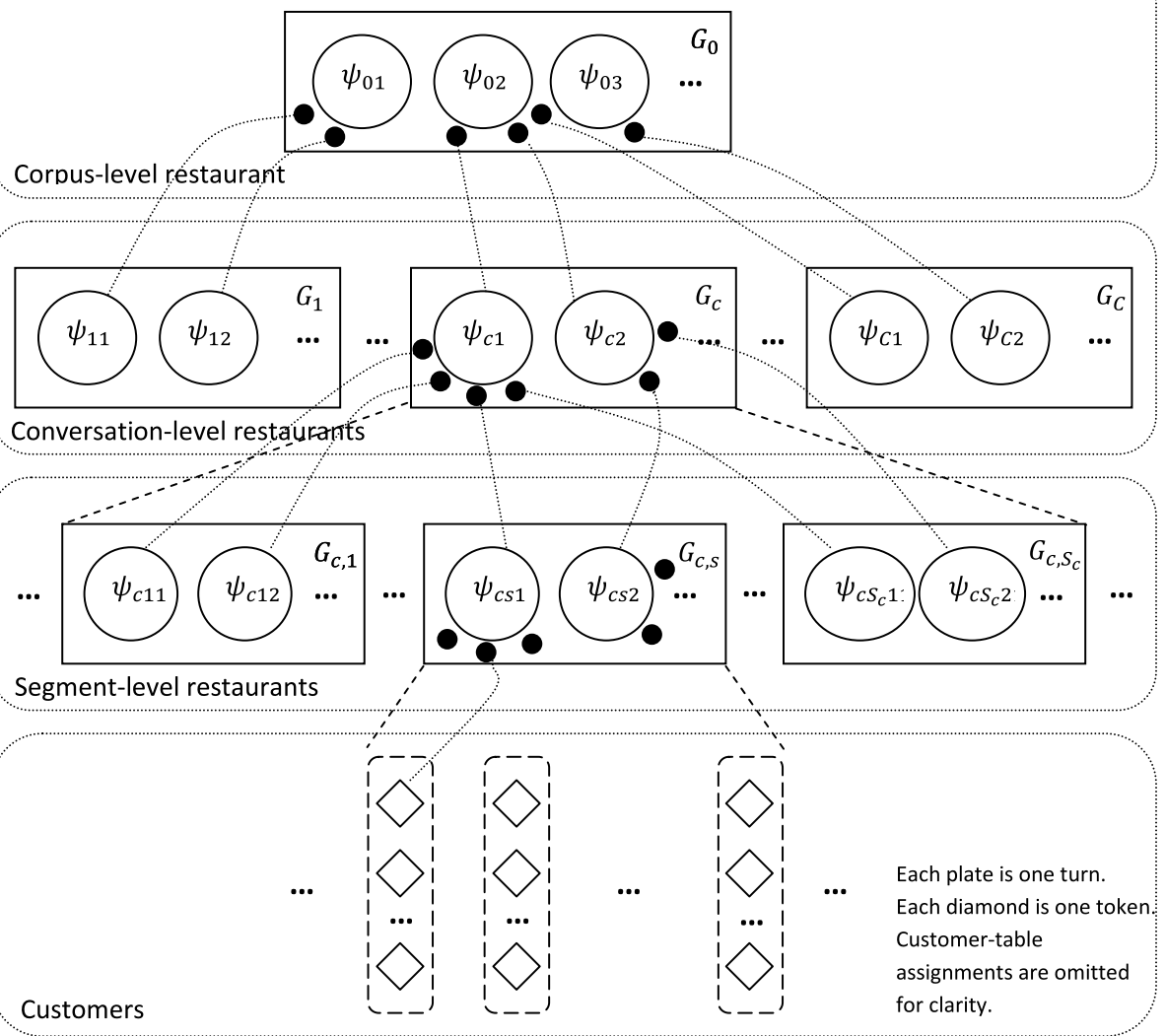

Fig. 3 Illustration of topic assignments in our inference algorithm. Each solid rectangle represents a restaurant (i.e., a topic distribution) and each circle represents a table (i.e., a topic). To assign token $n$ of turn $t$ in conversation $c$ to a table $z_{c, t, n}$ in the corpus-level restaurant, we need to sample a path assigning the token to a segment-level table, the segment-level table to a conversation-level table and the conversation-level table to a globally shared corpus-level table

To sample $z_{c, t, n}$, the index of the shared topic assigned to token $n$ of turn $t$ in conversation $c$, we need to sample the path assigning each word token to a segment-level table, each segment-level table to a conversation-level table and each conversation-level table to a shared dish. Before describing the sampling equations, we introduce notation denoting the counts:

- $N_{c, s, k}$ : number of tokens in segment $s$ in conversation $c$ assigned to dish $k$

- $N_{c, k}$ : number of segment-level tables in conversations $c$ assigned to dish $k$

- $N_{k}$ : number of conversation-level tables assigned to dish $k$.

Note that we use $k$ to index the global topics shared across the corpus, each of which corresponds to a dish in the corpus-level restaurant. In general, computing the exact values of these counts makes bookkeeping rather complicated. Since there might be multiple tables at a lower-level restaurant assigned to the same table at the higher-level restaurant, to compute the correct counts, we need to sum the number of customers over all these tables. For example, in Fig. 3, since both $\psi_{c, 1}$ and $\psi_{c, 2}$ are assigned to $\psi_{0,2}$ (i.e., $k=2$ ), to compute $N_{c, k}$ we 


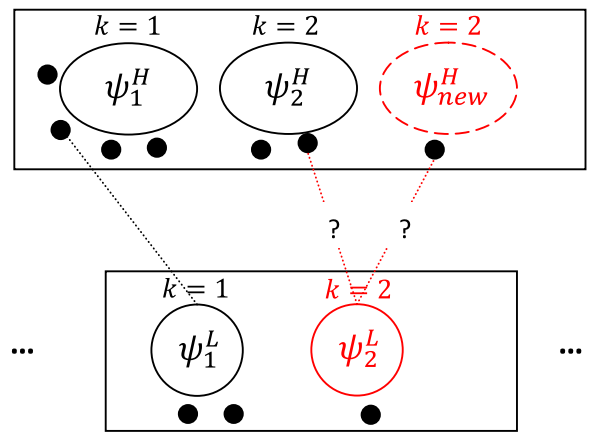

Fig. 4 Illustration of minimal path assumption. This figure shows an example of the seating assignments in a hierarchy of Chinese restaurants of a higher-level restaurant and a lower-level restaurant. Each table in the lower restaurant is assigned to a table in the higher restaurant and tables on the same path serve the same dish $k$. When sampling the assignment for table $\psi_{2}^{L}$ in the lower restaurant, given that dish $k=2$ is assigned to this table, there are two options for how the table in the higher restaurant could be selected. It could be an existing table $\psi_{2}^{H}$ or a new table $\psi_{n e w}^{H}$, both serving dish $k=2$. Under the minimal path assumption, it is always assigned to an existing table (if possible) and only assigned to a new table if there is no table with the given dish. In this case, the minimal path assumption will assign $\psi_{2}^{L}$ to $\psi_{2}^{H}$

have to sum over the number of customers currently assigned to $\psi_{c, 1}$ and $\psi_{c, 2}$ (which are 4 and 2 respectively in this example).

To mitigate this problem of bookkeeping and to speed up the sampling process, we use the minimal path assumption (Cowans 2006; Wallach 2008) to generate the path assignments. ${ }^{4}$ Under the minimal path assumption, a new table in a restaurant is created only when there is no table already serving the dish. In other words in a restaurant, there is at most one table serving a given dish. A more detailed example of the minimal path assumption is illustrated in Fig. 4. Using this assumption, in the example shown in Fig. 3, $\psi_{c, 1}$ and $\psi_{c, 2}$ will be merged together since they are both assigned to $\psi_{0,2}$.

Now that we have introduced our notations, the conditional distribution for $z_{c, t, n}$ is

$$
\begin{aligned}
& P\left(z_{c, t, n} \mid w_{c, t, n}, z^{-c, t, n}, \boldsymbol{w}^{-c, t, n}, \boldsymbol{l}, *\right) \\
& \quad \propto P\left(z_{c, t, n} \mid z^{-c, t, n}\right) P\left(w_{c, t, n} \mid z_{c, t, n}, \boldsymbol{w}^{-c, t, n}, \boldsymbol{l}, *\right)
\end{aligned}
$$

The first factor is the prior probability of assigning to a path according to the minimum path assumption (Wallach 2006, p. 60),

$$
P\left(z_{c, t, n}=k \mid z^{-c, t, n}\right) \propto \frac{N_{c, s_{t}, k}^{-c, t, n}+\alpha_{c} \frac{N_{c, k}^{-c, t, n}+\alpha_{0} \frac{N_{k}^{-c, t, n}+\alpha}{N^{-c, t, n}+\alpha}}{N_{c, \cdot}^{-c, t, n}+\alpha_{0}}}{N_{c, s_{t}, \cdot, n}^{-c, t,}+\alpha_{c}},
$$

\footnotetext{
${ }^{4}$ We also investigated using the maximal assumption and fully sampling assignments. We found the minimal path assumption worked as well as explicitly sampling seating assignments and that the maximal path assumption worked less well. Another, more complicated, sampling method is to sample the counts $N_{c, k}$ and $N_{k}$ according to their corresponding Antoniak distributions (Antoniak 1974), similar to the direct assignment sampling method described in Teh et al. (2006).
} 
where $K^{+}$is the current number of shared topics. ${ }^{5}$ Intuitively, (3) computes the probability of token $w_{c, t, n}$ being generated from a shared topic $k$. This probability is proportional to $N_{c, s_{t}, k}$ - the number of customers sitting at table serving dish $k$ at restaurant $G_{c, s_{t}}$, smoothed by the probability of generating this token from the table serving dish $k$ at the higher-level restaurant (i.e., restaurant $G_{c}$ ). This smoothing probability is computed in the same hierarchical manner until the top restaurant is reached, where the base distribution over topics is uniform and the probability of picking a topic is equal to $1 / K^{+}$. Equation (3) also captures the case where a table is empty; when the number of customers on that table is zero, the probability of generating the token from the corresponding topic relies entirely on the smoothing probability from the higher-level restaurant's table.

The second factor is the data likelihood. After integrating out all $\psi$ 's, we have

$$
P\left(w_{c, t, n}=w \mid z_{c, t, n}=k, \boldsymbol{w}^{-c, t, n}, \boldsymbol{l}, *\right) \propto \begin{cases}\frac{M_{k, w}^{-c, t, n}+\lambda}{M_{k, t, n}^{-c, t}+V \lambda}, & \text { if } k \text { exists } \\ \frac{1}{V}, & \text { if } k \text { is new }\end{cases}
$$

Here, $M_{k, w}$ denotes the number of times word type $w$ in the vocabulary is assigned to topic $k$; marginal counts are represented with $\cdot$ and $*$ represents all hyperparameters; $V$ is the size of the vocabulary, and the superscript ${ }^{-c, t, n}$ denotes the same counts excluding $w_{c, t, n}$.

\subsubsection{Sampling topic shift indicators}

Sampling the topic shift variable $l_{c, t}$ requires us to consider merging or splitting segments. We define the following notation:

- $\boldsymbol{k}_{c, t}$ : the shared topic indices of all tokens in turn $t$ of conversation $c$.

- $S_{a_{c, t}, x}$ : the number of times speaker $a_{c, t}$ is assigned the topic shift with value $x \in\{0,1\}$.

$-J_{c, s}^{x}$ : the number of topics in segment $s$ of conversation $c$ if $l_{c, t}=x$.

- $N_{c, s, j}^{x}$ : the number of tokens assigned to the segment-level topic $j$ when $l_{c, t}=x{ }^{6}$

Again, the superscript ${ }^{-c, t}$ is used to denote the exclusion of turn $t$ of conversation $c$ in the corresponding counts.

Recall that the topic shift is a binary variable. We use 0 to represent the "no shift" case, i.e. when the topic distribution is identical to that of the previous turn. We sample this assignment with the following probability:

$$
\begin{aligned}
& P\left(l_{c, t}=0 \mid \boldsymbol{l}^{-c, t}, \boldsymbol{w}, \boldsymbol{k}, \boldsymbol{a}, *\right) \\
& \propto \frac{S_{a_{c, t}, 0}^{-c, t}+\gamma}{S_{a_{c, t}, t}^{-c, t}+2 \gamma} \times \frac{\alpha_{c}^{J_{c, s_{t}}^{0}} \prod_{j=1}^{J_{c, s_{t}}^{0}}\left(N_{c, s_{t}, j}^{0}-1\right) !}{\prod_{x=1}^{N_{c, s_{t}, \cdot}^{0}}\left(x-1+\alpha_{c}\right)}
\end{aligned}
$$

In (5), the first factor is proportional to the probability of assigning a topic shift of value 0 to speaker $a_{c, t}$ and the second factor is proportional to the joint probability of all topics in segment $s_{t}$ of conversation $c$ when $l_{c, t}=0$. $^{7}$

\footnotetext{
${ }^{5}$ The superscript ${ }^{+}$is to denote that this number is unbounded and varies during the sampling process.

${ }^{6}$ Deterministically knowing the path assignments is the primary efficiency motivation for using the minimal path assumption. The alternative is to explicitly sample the path assignments, which is more complicated (for both notation and computation). This option is spelled out in full detail in the appendix.

${ }^{7}$ Refer to Gershman and Blei (2012) for a detailed derivation of this joint probability.
} 
Table 1 Summary of datasets detailing how many distinct speakers are present, how many distinct conversations are in the corpus, the annotations available, and the general content of the dataset. The $\dagger$ marks datasets we annotated

\begin{tabular}{lccll}
\hline Datasets & Speakers & Conversations & Annotations & Content \\
\hline ICSI Meetings & 60 & 75 & Topics & Engineering \\
2008 Debates & 9 & 4 & Topics & Politics \\
2012 Debates & 40 & 9 & None & Politics \\
Crossfire $\dagger$ & 2567 & 1134 & Influencer & Politics \\
Wikipedia discussions $\dagger$ & 604 & 1991 & Influencer & Varied \\
\hline
\end{tabular}

The other alternative is for the topic shift to be 1, which represents the introduction of a new distribution over topics inside an existing segment. The probability of sampling this assignment is:

$$
\begin{aligned}
& P\left(l_{c, t}=1 \mid \boldsymbol{l}^{-c, t}, \boldsymbol{w}, \boldsymbol{k}, \boldsymbol{a}, *\right) \\
& \quad \propto \frac{S_{a_{c, t}, 1}^{-c, t}+\gamma}{S_{a_{c, t}, \cdot,+}^{-c, t}+2 \gamma} \times\left(\frac{\alpha_{c}^{J_{c,\left(s_{t}-1\right)}^{1}} \prod_{j=1}^{J_{c,\left(s_{t}-1\right)}^{1}}\left(N_{c,\left(s_{t}-1\right), j}^{1}-1\right) !}{\prod_{x=1}^{N_{c,\left(s_{t}-1\right), \cdot}^{1}}\left(x-1+\alpha_{c}\right)} \frac{\alpha_{c}^{J_{c, s_{t}}^{1}} \prod_{j=1}^{J_{c, s}^{1}}\left(N_{c, s_{t} j}^{1}-1\right) !}{\prod_{x=1}^{N_{c, s}, \cdot}\left(x-1+\alpha_{c}\right)}\right)
\end{aligned}
$$

As above, the first factor in (6) is proportional to the probability of assigning a topic shift of value 1 to speaker $a_{c, t}$; the second factor in the big bracket is proportional to the joint distribution of the topics in segments $s_{t}-1$ and $s_{t}$. In this case, $l_{c, t}=1$ means splitting the current segment, which results in two joint probabilities for two segments.

\section{Data and annotations}

We validate our approach using five different datasets (Table 1). In this section, we describe the properties of each of the datasets and what information is available from the data. The datasets with interesting existing annotations typically are small and specialized. After validating our approach on simpler datasets, we move to larger datasets that we can explore qualitatively or by annotating them ourselves.

\subsection{Datasets}

We first describe the datasets that we use in our experiments. For all datasets, we tokenize texts using OpenNLP's tokenizer and remove common stopwords. ${ }^{8}$ After that, we remove turns that are very short since they do not contain much information content-wise and most likely there is no topic shift during these turns. We empirically remove turns that have fewer than 5 tokens after removing stopwords.

The ICSI meeting corpus The ICSI Meeting Corpus consists of 75 transcribed meetings at the International Computer Science Institute in Berkeley, California (Janin et al. 2003). Among these, 25 meetings were annotated with reference segmentations (Galley et al. 2003).

\footnotetext{
${ }^{8}$ http://opennlp.apache.org/.
} 
Table 2 Example turns from the annotated 2008 election debates (Boydstun et al. 2013). Each clause in a turn is coded with a Question Topic Code $\left(T_{Q}\right)$ and a Response Topic Code $\left(T_{R}\right)$. The topic codes $\left(T_{Q}\right.$ and $T_{R}$ ) are from the Policy Agendas Topics Codebook. In this example, the following topic codes are used: Macroeconomics (1), Housing \& Community Development (14), Government Operations (20)

\begin{tabular}{|c|c|c|c|c|}
\hline Speaker & Type & Turn clauses & $T_{Q}$ & $T_{R}$ \\
\hline Brokaw & $Q$ & $\begin{array}{l}\text { Sen. Obama, time for a discussion. I'm going to begin with you. Are } \\
\text { you saying to Mr. Clark and to the other members of the American } \\
\text { television audience that the American economy is going to get much } \\
\text { worse before it gets better and they ought to be prepared for that? }\end{array}$ & 1 & N/A \\
\hline \multirow[t]{2}{*}{ Obama } & $R$ & No, I am confident about the American economy. & 1 & 1 \\
\hline & & $\begin{array}{l}\text { But most importantly, we're going to have to help ordinary families be } \\
\text { able to stay in their homes, make sure that they can pay their bills, deal } \\
\text { with critical issues like health care and energy, and we're going to have } \\
\text { to change the culture in Washington so that lobbyists and special } \\
\text { interests aren't driving the process and your voices aren't being } \\
\text { drowned out. }\end{array}$ & 1 & 14 \\
\hline Brokaw & $Q$ & $\begin{array}{l}\text { Sen. McCain, in all candor, do you think the economy is going to get } \\
\text { worse before it gets better? }\end{array}$ & 1 & N/A \\
\hline \multirow[t]{3}{*}{ McCain } & $R$ & $\begin{array}{l}\text { I think if we act effectively, if we stabilize the housing market-which } \\
\text { I believe we can, }\end{array}$ & 1 & 14 \\
\hline & & $\begin{array}{l}\text { if we go out and buy up these bad loans, so that people can have a new } \\
\text { mortgage at the new value of their home. }\end{array}$ & 1 & 14 \\
\hline & & $\begin{array}{l}\text { I think if we get rid of the cronyism and special interest influence in } \\
\text { Washington so we can act more effectively. }\end{array}$ & 1 & 20 \\
\hline
\end{tabular}

Segmentations are binary, i.e., each point in the document is either a segment boundary or not, and on average each meeting has 8 segment boundaries. We use this dataset for evaluating topic segmentation (Sect. 5). After preprocessing, there are 60 unique speakers and the vocabulary contains 3346 non-stopword tokens.

The 2008 presidential election debates Our second dataset contains three annotated presidential debates between Barack Obama and John McCain and a vice presidential debate between Joe Biden and Sarah Palin (Boydstun et al. 2013). Each turn is one of two types: questions $(Q)$ from the moderator or responses $(R)$ from a candidate. Each clause in a turn is coded with a Question Topic Code $\left(T_{Q}\right)$ and a Response Topic Code $\left(T_{R}\right)$. Thus, a turn has a list of $T_{Q}$ 's and $T_{R}$ 's both of length equal to the number of clauses in the turn. Topics are from the Policy Agendas Topics Codebook, a widely used inventory containing codes for 19 major topics and 225 subtopics. ${ }^{9}$ Table 2 shows an example annotation.

To obtain reference segmentations in debates, we assign each turn a real value from 0 to 1 indicating how much a turn changes the topic. For a question-typed turn, the score is the fraction of clause topic codes not appearing in the previous turn; for response-typed turns, the score is the fraction of clause topic codes that do not appear in the corresponding question. This results in a set of non-binary reference segmentations. For evaluation metrics that require binary segmentations, we create a binary segmentation by labeling a turn as a segment boundary if the computed score is 1 . This threshold is chosen to include only true segment boundaries. After preprocessing, this dataset contains 9 unique speakers and the vocabulary contains 1,761 non-stopword tokens.

\footnotetext{
${ }^{9}$ http://www.policyagendas.org/page/topic-codebook.
} 
Table 3 List of the 9 Republican Party presidential debates used

\begin{tabular}{llll}
\hline Date & Place & Sponsor & Participants \\
\hline 13 Jun. 2011 & Goffstown, NH & CNN & $\begin{array}{l}\text { Bachmann, Cain, Gingrich, Paul, Pawlenty, Romney, } \\
\text { Santorum }\end{array}$ \\
12 Sep. 2011 & Tampa, FL & CNN & $\begin{array}{l}\text { Bachmann, Cain, Gingrich, Huntsman, Paul, Perry, } \\
\text { Romney, Santorum }\end{array}$ \\
18 Oct. 2011 & Las Vegas, NV & CNN & $\begin{array}{l}\text { Bachmann, Cain, Gingrich, Paul, Perry, Romney, } \\
\text { Santorum }\end{array}$ \\
09 Nov. 2011 & Rochester, MI & CNBC & $\begin{array}{l}\text { Bachmann, Cain, Gingrich, Huntsman, Paul, Perry, } \\
\text { Romney, Santorum }\end{array}$ \\
22 Nov. 2011 & Washington, DC & CNN & $\begin{array}{l}\text { Bachmann, Cain, Gingrich, Huntsman, Paul, Perry, } \\
\text { Romney, Santorum }\end{array}$ \\
19 Jan. 2012 & Charleston, SC & CNN & $\begin{array}{l}\text { Gingrich, Paul, Romney, Santorum } \\
\text { 23 Jan. 2012 }\end{array}$ \\
26 Jan. 2012 & Tampa, FL & NBC & Gingrich, Paul, Romney, Santorum \\
22 Feb. 2012 & Mesa, AZ & CNN & $\begin{array}{l}\text { Gingrich, Paul, Romney, Santorum } \\
\text { Gingrich, Paul, Romney, Santorum }\end{array}$ \\
\hline
\end{tabular}

The 2012 republican primary debates We also downloaded nine transcripts in the 2012 Republican Party presidential debates, whose information is shown in Table 3. Since the transcripts are pulled from different sources, we perform a simple entity resolution step using edit distance to merge duplicate participants' names. For example, "ROMNEY", "MiTT RoMney" are resolved into "Romney"; "PAUL", "ReP. PAUL", "REPRESEnTATIVE Ron PAUL R-TX" are resolved into "PAUL" etc. We also merge anonymous participants such as "Unidentified Female", "Unidentified MALE", "Question”, "UnKNOWN" etc. into a single participant named "AUDIENCE". After preprocessing, there are 40 unique participants in these 9 debates including candidates, moderators and audience members. This dataset is not annotated and we only use it for qualitative evaluation.

CNN's crossfire Crossfire was a weekly U.S. television "talking heads" program engineered to incite heated arguments (hence the name). Each episode features two recurring hosts, two guests, and clips from the week's news. Our Crossfire dataset contains 1134 transcribed episodes aired between 2000 and 2004. ${ }^{10}$ There are 2567 unique speakers and the vocabulary size is 16,791 . Unlike the previous two datasets, Crossfire does not have explicit topic segmentations, so we use it to explore speaker-specific characteristics (Sect. 6.2).

Wikipedia discussions Each article on Wikipedia has a related discussion page so that the individuals writing and editing the article can discuss the content, editorial decisions, and the application of Wikipedia policies (Butler et al. 2008). Unlike the other situations, Wikipedia discussions are not spoken conversations that have been transcribed. Instead, these conversations are written asynchronously.

However, Wikipedia discussions have much of the same properties as our other corpora. Contributors have different levels of responsibility and prestige, and many contributors are actively working to persuade the group to accept their proposed policies (for an example, see Table 4), other contributors are attempting to maintain civility, and other contributors are attacking their ostensible collaborators.

\footnotetext{
${ }^{10}$ http://www.cs.umd.edu/ vietan/topicshift/data.zip.
} 
Table 4 Example of a Wikipedia discussion in our dataset

A: The current lead sentence has been agreed upon by many-I know, I was embroiled in the huge debate that developed into the current lead. However, the sentence is still kinda awkward-even though it captures the broader essence of evolutionary theory. I would like to propose an alternate (below), because there is a problem with the way that the term change is used, as Kirk J. Fitzhugh has noted: "Change is not the pertinent quality of interest in evolution". Hence: Evolution is the gradual departure across successive generations in the constituency of the inherited characteristics of organisms in biological populations.

B: No thank you, this is just more obscurantism.

A: It's wp:V, not obscurantism, consistent with the history of the science. Not much thought goes into conceiving that "Evolution is change", but if you are asked to think past this and call it obscurantism in your critique, it is a strange response. Obscurantism: "is the practice of deliberately preventing the facts or the full details of some matter from becoming known"-ironic that this applies more aptly to your rejection.

B: Your obsession with providing the most scientifically accurate and current definition of evolution prevents the average reader from having a chance at understanding this article. That is obscurantism. It is not WPV, because that definition is not by a longshot the most commonly used, and specifically it is entirely unsuited for works meant to be read by lay readers.

C: This is a general encyclopedia, not a graduate level evolutionary biology course. Keeping it simple so that people can understand what we write without having an advanced degree is a good thing. So no, let's keep the lead as is.

Unlike spoken conversations, Wikipedia discussions lack social norms that prevent an individual from writing as often or as much as they want. This makes common techniques such as counting turns or turn lengths less helpful measures to discover who influencers are.

\subsection{Influencer annotation}

Our goal is to discover who are the influencers in these discussions. To assess our ability to discover influencers, we annotated randomly selected documents from both the Wikipedia and Crossfire datasets. This process proceeded as follows. First, we followed the annotation guidelines for influencers proposed by Bender et al. (2011) for Wikipedia discussion. A discussant is considered an influencer if he or she initiated a topic shift that steered the conversation in a different direction, convinced others to agree to a certain viewpoint, or used an authoritative voice that caused others to defer to or reference that person's expertise. A discussant is not identified as an influencer if he or she merely initiated a topic at the start of a conversation, did not garner any support from others for the points he or she made, or was not recognized by others as speaking with authority. After annotating an initial set of documents, we revised our annotation guidelines and retrained two independent annotators until we reached an intercoder reliability Cohen's Kappa (Artstein and Poesio 2008) of $0.8 .^{11}$

Wikipedia discussions Coders first learned to annotate transcripts using Wikipedia discussion data. The two coders annotated over 400 English Wikipedia discussion transcripts for

\footnotetext{
${ }^{11}$ Kappa was measured based on whether the two annotators agreed on (a) whether there was an influencer, (b) who the primary influencer was, and (c) if there was a secondary influencer. When discrepancies occurred between the annotators, they were resolved through discussion between the annotators and with the supervising researcher. So decisions were not "yes or no" about each speaker; instead, they were about whether or not there was an influencer in each overall interaction, and if so, who the primary and secondary influencers were in a particular interaction.
} 
influencer in batches of 20 to 30 transcripts each week. For the English transcripts, each coder annotated the transcripts independently, then annotations were compared for agreement; any discrepancies in the annotations were resolved through discussion of how to apply the coding scheme. After the first four sets of 20 to 30 transcripts, the coders were able to code the transcripts with acceptable intercoder reliability (Cohen's Kappa $>0.8$ ). Once the coders reached acceptable intercoder reliability for two sets of English data in a row, the coders began independently coding the remaining set of transcripts. Intercoder reliability was maintained at an acceptable level (Cohen's Kappa > 0.8) for the English transcripts over the subsequent weeks of coding.

Crossfire We then turned our attention to the Crossfire dataset. We split each Crossfire episode into smaller segments using the "COMMERCIAL_BREAK" tags and use each segment as a unit of conversation. The same two coders annotated the Crossfire data. To prepare for annotating the Crossfire interactions, the coders both annotated the same set of 20 interactions. First the intercoder reliability Cohen's Kappa was calculated for the agreement between the coders, then any disagreements between the coders were resolved through discussion about the discrepant annotations. The first set of 20 transcripts was coded with a Cohen's Kappa of 0.65 (before discussion). This procedure was repeated twice; each time the coders jointly annotated 20 transcripts, reliability was calculated, and any discrepancies were resolved through discussion. The third set achieved an acceptable Cohen's Kappa of 0.8 . The remaining transcripts were then split and annotated separately by the two coders. In all, 105 Crossfire episode segments were annotated. An annotation guideline for Crossfire is included in the Appendix B.

\section{Evaluating topic segmentation}

In this section, we examine how well SITS can identify when new topics are introduced, i.e., how well it can segment conversations. We discuss metrics for evaluating an algorithm's segmentation relative to a gold annotation, describe our experimental setup, and report those results.

\subsection{Experiment setups}

Evaluation metrics To evaluate the performance on topic segmentation, we use $P_{k}$ (Beeferman et al. 1999) and WindowDiff (WD) (Pevzner and Hearst 2002). Both metrics measure the probability that two points in a document will be incorrectly separated by a segment boundary. Both techniques consider all windows of size $k$ in the document and count whether the two endpoints of the window are (im)properly segmented against the gold segmentation. More formally, given a reference segmentation $\mathcal{R}$ and a hypothesized segmentation $\mathcal{H}$, the value of $P_{k}$ for a given window size $k$ is defined as follow:

$$
P_{k}=\frac{\sum_{i=1}^{N-k} \delta_{\mathcal{H}}(i, i+k) \oplus \delta_{\mathcal{R}}(i, i+k)}{N-k}
$$

where $\delta_{\mathcal{X}}(i, j)$ is 1 if the segmentation $\mathcal{X}$ assigns $i$ and $j$ to the same segment and 0 otherwise; $\oplus$ denotes the XOR operator; $N$ is the number of candidate boundaries.

WD improves $P_{k}$ by considering how many boundaries lie between two points in the document, instead of just looking at whether the two points are separated or not. WD of size 
$k$ between two segmentations $\mathcal{H}$ and $\mathcal{R}$ is defined as:

$$
\mathrm{WD}=\frac{\sum_{i=1}^{N-k}\left[\left|b_{\mathcal{H}}(i, i+k)-b_{\mathcal{R}}(i, i+k)\right|>0\right]}{N-k}
$$

where $b_{\mathcal{X}}(i, j)$ counts the number of boundaries that the segmentation $\mathcal{X}$ puts between two points $i$ and $j$.

However, these metrics have a major drawback. They require both hypothesized and reference segmentations to be binary. Many algorithms (e.g., probabilistic approaches) give non-binary segmentations where candidate boundaries have real-valued scores (e.g., probability or confidence). Thus, evaluation requires arbitrary thresholding to binarize soft scores. In previous work, to be fair for all methods, thresholds are usually set so that the number of segments is equal to a predefined value (Purver et al. 2006; Galley et al. 2003). In practice, this value is usually unknown.

To overcome these limitations, we also use $\widehat{E M D}$ (Pele and Werman 2008), a variant of the Earth Mover's Distance (EMD). Originally proposed by Rubner et al. (2000), EMD is a metric that measures the distance between two normalized histograms. Intuitively, it measures the minimal cost that must be paid to transform one histogram into the other. EMDis a true metric only when the two histograms are normalized (e.g., two probability distributions). $\widehat{E M D}$ relaxes this restriction to define a metric for non-normalized histograms by adding or subtracting masses so that both histograms are of equal size.

Applied to our segmentation problem, each segmentation can be considered a histogram where each candidate boundary point corresponds to a bin. The probability of each point being a boundary is the mass of the corresponding bin. We use $|i-j|$ as the ground distance between two points $i$ and $j .{ }^{12}$ To compute $\widehat{\text { EMD }}$ we use the FastEMD implementation (Pele and Werman 2009).

Experimental methods We applied the following methods to discover topic segmentations in a conversation:

- TextTiling (Hearst 1997) is one of the earliest and most widely used general-purpose topic segmentation algorithms, sliding a fixed-width window to detect major changes in lexical similarity.

- P-NoSpeaker-single: parametric version of SITS without speaker identity, run individually on each conversation (Purver et al. 2006).

- P-NoSpeaker-all: parametric version of SITS without speaker identity run on all conversations.

- P-SITS: the parametric version of SITS with speaker identity run on all conversations.

- NP-HMM: the HMM-based nonparametric model with speaker identity. This model uses the same assumption as the Sticky HDP-HMM (Fox et al. 2008), where a single topic is associated with each turn.

- NP-SITS: the nonparametric version of SITS with speaker identity run on all conversations.

Parameter settings and implementation In our experiment, all parameters of TextTiling are the same as in Hearst (1997). For statistical models, Gibbs sampling with 10 randomly

\footnotetext{
${ }^{12}$ The ground distance is the distance between two bins in a histogram. Please refer to Pele and Werman (2008) for a more formal definition of $\widehat{\mathrm{EMD}}$.
} 
Table 5 Results on the topic segmentation task. Lower is better. The parameter $k$ is the window size of the metrics $P_{k}$ and WindowDiff chosen to replicate previous results

\begin{tabular}{|c|c|c|c|c|c|c|c|c|}
\hline & \multirow[t]{2}{*}{ Model } & \multirow[t]{2}{*}{$\overline{E M D}$} & \multicolumn{3}{|l|}{$P_{k}$} & \multicolumn{3}{|c|}{ WindowDiff } \\
\hline & & & $k=5$ & 10 & 15 & $k=5$ & 10 & 15 \\
\hline \multirow[t]{6}{*}{ ICSI } & TextTiling & 2.507 & .289 & .388 & .451 & .318 & .477 & .561 \\
\hline & P-NoSpeaker-single & 1.949 & .222 & .283 & .342 & .269 & .393 & .485 \\
\hline & P-NoSpeaker-all & 1.935 & 207 & .279 & .335 & .253 & .371 & .468 \\
\hline & P-SITS & 1.807 & 211 & .251 & .289 & .256 & .363 & .434 \\
\hline & NP-HMM & 2.189 & .232 & .257 & .263 & .267 & .377 & .444 \\
\hline & NP-SITS & 2.126 & .228 & .253 & .259 & .262 & .372 & .440 \\
\hline \multirow[t]{6}{*}{2008 Debates } & TextTiling & 2.821 & .433 & .548 & .633 & .534 & 674 & .760 \\
\hline & P-NoSpeaker-single & 2.822 & .426 & .543 & .653 & .482 & .650 & .756 \\
\hline & P-NoSpeaker-all & 2.712 & .411 & .522 & .589 & .479 & .644 & .745 \\
\hline & P-SITS & 2.269 & .380 & .405 & .402 & .482 & .625 & .719 \\
\hline & NP-HMM & 2.132 & .362 & .348 & .323 & .486 & .629 & .723 \\
\hline & NP-SITS & 1.813 & .332 & .269 & .231 & .470 & .600 & 692 \\
\hline
\end{tabular}

initialized chains is used. Initial hyperparameter values are sampled from $U(0,1)$ to favor sparsity; statistics are collected after 500 burn-in iterations with a lag of 25 iterations over a total of 5000 iterations; and slice sampling (Neal 2003) optimizes hyperparameters. Parametric models are run with 25, 50 and 100 topics and the best results (averaged over 10 chains) are reported.

\subsection{Results and analysis}

Table 5 shows the performance of various models on the topic segmentation problem, using the ICSI corpus and the 2008 debates.

Consistent with previous results in the literature, probabilistic models outperform TextTiling. In addition, among the probabilistic models, the models that had access to speaker information consistently segment better than those lacking such information. Furthermore, NP-SITS outperforms NP-HMM in both experiments, suggesting that using a distribution over topics for turns is better than using a single topic. This is consistent with the parametric models in Purver et al. (2006).

The contribution of speaker identity seems more valuable in the debate setting. Debates are characterized by strong rewards for setting the agenda; dodging a question or moving the debate toward an opponent's weakness can be useful strategies (Boydstun et al. 2013). In contrast, meetings (particularly low-stakes ICSI meetings, technical discussions in R\&D group) tend to have pragmatic rather than strategic topic shifts. In addition, agenda-setting roles are clearer in formal debates; a moderator is tasked with setting the agenda and ensuring the conversation does not wander too much.

The nonparametric model does best on the smaller debate dataset. We suspect that an evaluation that directly accessed the topic quality, either via prediction (Teh et al. 2006) or interpretability (Chang et al. 2009b) would favor the nonparametric model more. 
Fig. 5 Topic shift tendency $\pi$ of speakers in the 2008 Presidential Election Debates (larger means greater tendency). IFILL was the moderator in the vice presidential debate between BIDEN and PALIN; BROKAW, LEHRER and SCHIEFFER were the moderators in the three presidential debates between OBAMA and MCCAIN; QUESTION collectively refers to questions from the audiences

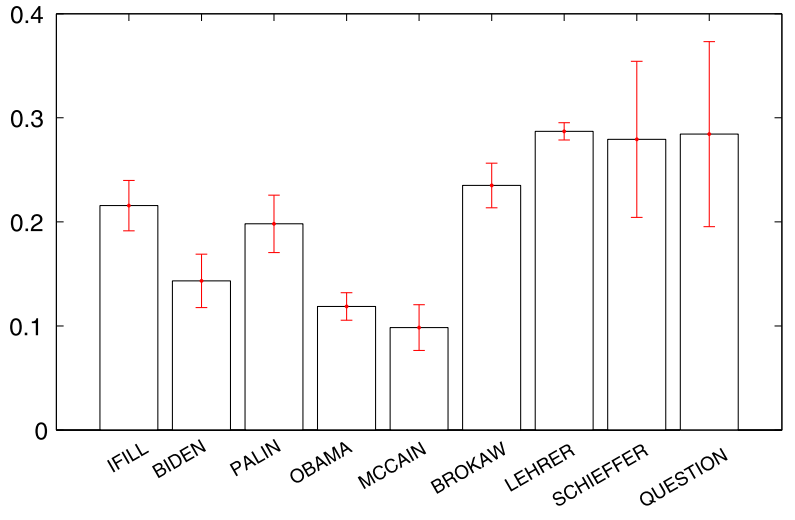

\section{Evaluating topic control}

In this section, we focus on the ability of SITS to capture the extent to which individual speakers affect topic shifts in conversations. Recall that SITS associates with each speaker a topic shift tendency $\pi$ that represents the probability of changing the topic in the conversation. While topic segmentation is a well studied problem, hence the evaluation in Sect. 5, there are no established quantitative measurements of an individual's ability to control a conversation. To evaluate whether the tendency is capturing meaningful characteristics of speakers, we look qualitatively at the behavior of the model.

\subsection{8 election debates}

To obtain a posterior estimate of $\pi$ (Fig. 5) we create 10 chains with hyperparameters sampled from the uniform distribution $U(0,1)$ and average $\pi$ over 10 chains (as described in Sect. 5.1). In these debates, Ifill is the moderator of the debate between Biden and Palin; Brokaw, Lehrer and Schieffer are the three moderators of the three debates between Obama and McCain. Here "Question" denotes questions from audiences in "town hall" debate. The role of this "speaker" can be considered equivalent to the debate moderator.

The topic shift tendencies of moderators are generally much higher than for candidates. In the three debates between Obama and McCain, the moderators-Brokaw, Lehrer and Schieffer-have significantly higher scores than both candidates. This is a useful reality check, since in a debate the moderators are the ones asking questions and literally controlling the topical focus. Similarly, the "Question" speaker had a relatively high variance, consistent with that "participant" in the model as an amalgamation of many distinct speakers.

Interestingly, however, in the vice-presidential debate, the score of moderator Ifill is higher than the candidates' scores only by a small margin, and it is indistinguishable from the degree of topic control displayed by Palin. Qualitatively, the assessment of the model is consistent with widespread perceptions and media commentary at the time that characterized Ifill as a weak moderator. For example, Harper's Magazine's Horton (2008) discusses the context of the vice-presidential debate, in particular the McCain campaign's characterization of Ifill as a biased moderator because she "was about to publish a book entitled The Breakthrough that discusses Barack Obama, and a number of other black politicians, achieving national prominence". According to Horton:

First, the charges against Ifill would lead to her being extremely passive in her questioning of Palin and permissive in her moderating the debate. Second, the charge of 
bias against Ifill would enable Palin to simply skirt any questions she felt uncomfortable answering and go directly to a pre-rehearsed and nonresponsive talking point. This strategy succeeded on both points.

Similarly, Fallows (2008) of The Atlantic included the following in his "quick guide" remarks on the debate:

Ifill, moderator: Terrible. Yes, she was constrained by the agreed debate rules. But she gave not the slightest sign of chafing against them or looking for ways to follow up the many unanswered questions or self-contradictory answers. This was the big news of the evening ...

Palin: "Beat expectations." In every single answer, she was obviously trying to fit the talking points she had learned to the air time she had to fill, knowing she could do so with impunity from the moderator.

That said, our quantitative modeling of topic shift tendency suggests that all candidates managed to succeed at some points in setting and controlling the topic of conversation in the debates. In the presidential debates, our model gives Obama a slightly higher score than McCain, consistent with social science claims that Obama had the lead in setting the agenda over McCain (Boydstun et al. 2013). Table 6 shows some examples of SITS-detected topic shifts.

\subsection{Crossfire}

The Crossfire dataset has many more speakers than the presidential and vice-presidential debates. This allows us to examine more closely what we can learn about speakers' topic shift tendency and ask additional questions; for example, assuming that changing the topic is useful for a speaker, how can we characterize who does so effectively? In our analysis, we take advantage of properties of the Crossfire data to examine the relationship between topic shift tendency, social roles, and political ideology.

In order to focus on frequent speakers, we filter out speakers with fewer than 30 turns. Most speakers have relatively small $\pi$, with the mode around 0.3 . There are, however, speakers with very high topic shift tendencies. Table 7 shows the speakers having the highest values according to SITS.

We find that there are three general patterns for who influences the course of a conversation in Crossfire. First, there are structural "speakers" that the show uses to frame and propose new topics. These are audience questions, news clips (e.g. many of Gore's and Bush's turns from 2000), and voiceovers. That SITS is able to recover these is reassuring, similar to what it has to say about moderators in the 2008 debates. Second, the stable of regular hosts receives high topic shift tendencies, which is again reasonable given their experience with the format and ostensible moderation roles (though in practice they also stoke lively discussion).

The third category is more interesting. The remaining non-hosts with high topic shift tendency appear to be relative moderates on the political spectrum:

- John Kasich, one of few Republicans to support the assault weapons ban and who was elected in 2010 as the governor of Ohio, a swing state

- Christine Todd Whitman, former Republican governor of New Jersey, a very Democratic state

- John McCain, who before 2008 was known as a "maverick" for working with Democrats (e.g. Russ Feingold). 
Table 6 Example of turns designated as a topic shift by SITS. We chose turns to highlight speakers with high topic shift tendency $\pi$. Some keywords are manually italicized to highlight the topics discussed

Previous turn Turn detected as shifting topic

2008 Debates Dataset BIDEN: Well, mortgage-holders didn't pay the price [...] Barack Obama pointed out two years ago that there was a subprime mortgage [...]

PALIN: Your question to him was whether he supported gay marriage and my answer is the same as his and it is that I do not.

MCCAIN: I think that Joe Biden is qualified in many respects....

IFILL: So, Governor, as vice president, there's nothing that you have promised [...] that you wouldn't take off the table because of this financial crisis we're in?

Crossfire Dataset

PRESS: But what do you say, governor, to Governor Bush and [...] your party who would let politicians and not medical scientists decide what drugs are distributed [...]

WEXLER: [...] They need a Medicare prescription drug plan [...] Talk about schools, [...] Al Gore has got a real plan. George Bush offers us vouchers. Talk about the environment. [...] Al Gore is right on in terms of the majority of Americans, but George Bush [...]

PRESS: Senator, Senator Breaux mentioned that it's President Bush's aim to start on education [...] [McCain] [...] said he was going to do introduce the legislation the first day of the first week of the new administration. [...]
PALIN: That is not so, but because that's just a quick answer, I want to talk about, again, my record on energy ... When we talk about energy, we need to consider the need to do all that we can to allow this nation to become energy independent $[\ldots]$

IFILL: Wonderful. You agree. On that note, let's move to foreign policy. You both have sons who are in Iraq or on their way to Iraq. You, Governor Palin, have said that you would like to see a real clear plan for an exit strategy. [...]

SCHIEFFER: [...] Let's talk about energy and climate control. Every president since Nixon has said what both of you [...]

BIDEN: Again, let me-let's talk about those tax breaks. [Obama] voted for an energy bill because, for the first time, it had real support for alternative energy. [...] on eliminating the tax breaks for the oil companies, Barack Obama voted to eliminate them. [...]

WHITMAN: Well I disagree with them on this particular issues [...] that's important to me that George Bush stands for education of our children [...] I care about tax policy, I care about the environment. I care about all the issues where he has a proven record in Texas [...]

KASICH: [...] I want to talk about choice. [...] George Bush believes that, if schools fail, parents ought to have a right to get their kids out of those schools and give them a chance and an opportunity for success. Gore says "no way" [...] Social Security. George Bush says [...] direct it the way federal employees do [...] Al Gore says "No way" [...] That's real choice. That's real bottom-up, not a bureaucratic approach, the way we run this country.

MCCAIN: After one of closest elections in our nation's history, there is one thing the American people are unanimous about. They want their government back. We can do that by ridding politics of large, unregulated contributions that give special interests a seat at the table while average Americans are stuck in the back of the room. 
Table 7 Top speakers by topic shift tendencies from our Crossfire dataset. We mark hosts ( $\dagger$ ) and "speakers" who often (but not always) appeared in video clips ( $\$$ ). ANNOUNCER makes announcements at the beginning and at the end of each show; NARRATOR narrates video clips; MALE and FEMALE refer to unidentified male and female respectively; QUESTION collectively refers to questions from the audience across different shows. Apart from those groups, speakers with the highest tendency were political moderates

\begin{tabular}{|c|c|c|c|c|c|}
\hline Rank & Speaker & $\pi$ & Rank & Speaker & $\pi$ \\
\hline 1 & ANNOUNCER & .884 & 10 & JOHN KASICH & .570 \\
\hline 2 & MALE & .876 & 11 & JAMES CARVILLE ${ }^{\dagger}$ & .550 \\
\hline 3 & QUESTION & .755 & 12 & TUCKER CARLSON $^{\dagger}$ & .550 \\
\hline 4 & GEORGE W. BusH ${ }^{\ddagger}$ & .751 & 13 & PAUL BEGALA ${ }^{\dagger}$ & .545 \\
\hline 5 & BILL PRESS $^{\dagger}$ & .651 & 14 & CHRISTINE T. WHITMAN & .533 \\
\hline 6 & FEMALE & .650 & 15 & TERRY MCAULIFFE & .529 \\
\hline 7 & AL GORE ${ }^{\ddagger}$ & .650 & 16 & MARY MATALIN ${ }^{\dagger}$ & .527 \\
\hline 8 & NARRATOR ${ }^{\ddagger}$ & .642 & 17 & JOHN MCCAIN & .524 \\
\hline 9 & ROBERT NOVAK ${ }^{\dagger}$ & .587 & 18 & ARI FLEISCHER & .522 \\
\hline
\end{tabular}

Although these observations are at best preliminary and require further investigation, we would conjecture that in Crossfire's highly polarized context, it was the political moderates who pushed back, exerting more control over the agenda of the discussion, rather than going along with the topical progression and framing as posed by the show's organizers. Table 6 shows several detected topic shifts from these speakers. In two of these examples, McCain and Whitman are Republicans disagreeing with President Bush. In the other, Kasich is defending a Republican plan (school vouchers) popular with traditional Democratic constituencies.

\subsection{Republican primary debates}

As another qualitative data point, we include in Fig. 6 the model's topic shift tendency scores for a subset of nine 2012 Republican primary debates. Although we do not have objective measures to compare against, nor clearly stated contemporary commentary as in the case of Ifill's performance as moderator, we would argue that the model displays quite reasonable face validity in the context of the Republican race.

For example, among the Republican candidates, Ron Paul is known for tight focus on a discrete set of arguments associated with his position that "the proper role for government in America is to provide national defense, a court system for civil disputes, a criminal justice system for acts of force and fraud, and little else" (Paul 2007), often regardless of the specific question that was asked. Similarly, Rick Santorum's performance in the primary debates tended to include strong rhetoric on social issues. In contrast, Mitt Romney tended to be less aggressive in his responses, arguably playing things safer in a way that was consistent with his general position throughout the primaries as the front-runner.

\section{Detecting influencers in conversations}

\subsection{Computational methods for influencer detection}

In this section, we turn to the direct application and validation of the model in detecting influencers in conversations. Even though influence in conversations has been studied for decades 


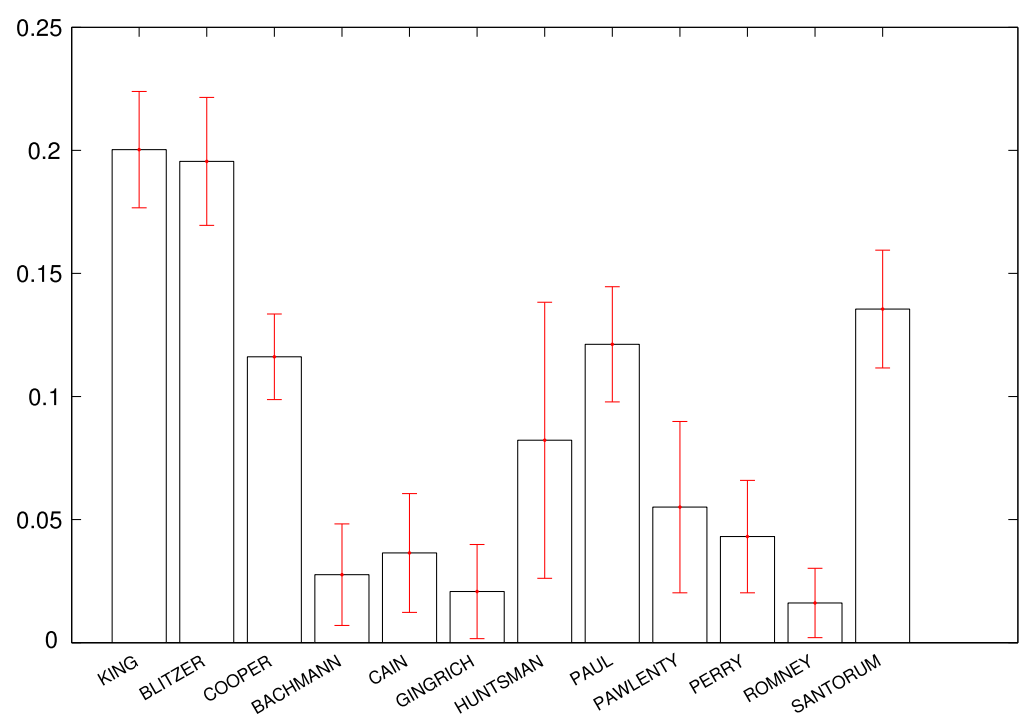

Fig. 6 Topic shift tendency $\pi$ of speakers in the 2012 Republican Primary Debates (larger means greater tendency). KING, BLITZER and COOPER are moderators in these debates; the rest are candidates

in communication and social psychology, computational methods have only emerged in recent years, thanks to improvements in both quantity and quality of conversational data. As one example, an early computational model to quantify influence between conversational participants (Basu et al. 2001) modeled interactions among a conversational group in a multi-sensor lounge room where people played interactive debating games. In these games, each participant can be in two states: speaker or silent. The model equates each participant with a Markov model. Each participant is allowed to be in either speaking state or silent state at each time step and the transition from one state to another of an individual is influenced by other participants' states. This allows the model to capture pair-wise interactions among participants in the conversation. Zhang et al. (2005) then extended the work by proposing a model with two-level structure: the participant level, representing the actions of individual participants, and the group level, representing group-level actions. In this setting, the influence of each participant on the actions of the whole group is explicitly captured by the model. These models use expensive features such as prosody and visual cues.

Another popular approach is to treat influencer detection as a supervised classification problem that separates influential individuals from non-influential ones. Rienks and Heylen (2005) focus on extracting a set of structural features that can predict participants' involvement using Support Vector Machines (Cortes and Vapnik 1995, SVM). Later, Rienks et al. (2006) improved their previous work by extending the set of features to include features capturing topic changes as well as those derived from audio and speech. Again, we do not use any features extracted from audio or visual data, which makes our approach more generalizable. The two most relevant and most useful features extracted from the meeting textual transcripts are number of turns and length of turns, which we use as the baseline in our experiments described in Sect. 7.2. Biran et al. (2012) also follow a similar approach to detecting influencers in written online conversations by extracting features to capture different conversational behaviors such as persuasion, agreement/disagreement and dialog patterns.

In this paper, we are interested in determining who are the influencers in a conversation using only the conversation transcripts. We tackle this problem by using an unsupervised 
ranking approach. It is worth mentioning that, even though we are focused on studying how conversational influence expressed in textual data, there has also been a body of work approaching this problem by studying audio data (Hung et al. 2011), visual data (Otsuka et al. 2006) and both audio-visual activity cues (Jayagopi et al. 2009; Aran and Gatica-Perez 2010).

Our main purpose in this experimentation is to assess how effective SITS can be in detecting influencers in conversations, especially in comparison with methods based on structural patterns of conversations. We focus on the influencer detection problem: given a speaker in a multi-party conversation, predict whether the speaker is influential. In the remaining of this section, we describe in details the approach we take, the experimental setups, and the results.

\subsection{Influencer detection problem}

The influencer detection problem can be tackled using different methods that can be broadly classified into classification and ranking approaches. Most previous work follows the classification approach, in which different sets of features are proposed and a classifier is used (Rienks and Heylen 2005; Rienks et al. 2006; Biran et al. 2012). In this paper, we follow the ranking approach.

The ranking approach allows us to focus on individual functions that take a set of individuals and produce an ordering over those individuals from most influential to least influential. The function that produces this ordering is called a ranking method. More specifically, given a speaker $a$ in a conversation $c$, each ranking method will provide an influence score $\mathcal{I}_{a, c}$ that indicates how influential speaker $a$ is in conversation $c$. We emphasize that, unlike most classification approaches (Rienks and Heylen 2005; Rienks et al. 2006; Biran et al. 2012), the ranking approach we are focusing on is entirely unsupervised and thus requires no training data.

The ranking approach has a straightforward connection to the classification approach, as each ranking function can be turned into a feature in the supervised classification framework. However, viewing the ranking methods (features) independently allows us to compare and interpret the effectiveness of each feature in isolation. This is useful as an evaluation method because it is independent of the choice of classifier and is less sensitive to the size of training data, which is often a limiting factor in computational social science.

We consider two sets of ranking methods: (1) structure-based methods, which use structural features and (2) topic-change-based methods, which use features extracted from the outputs of SITS.

Structure-based methods score each instance based on features extracted from the structure of the conversation. As defined in Sect. 2, we use $T_{c}$ to denote the number of turns in conversation $c ; a_{c, t}$ to denote the speaker that utters turn $t$ in conversation $c$; and $N_{c, t}$ to denote the number of tokens in turn $t$ in conversation $c$.

1. Number of turns: assumes that the more turns a speaker has during a conversation, the more influential he or she is. The influence score of this method is

$$
\mathcal{I}_{a, c}=\left|\left\{t \in\left[1, T_{c}\right]: a_{c, t}=a\right\}\right|
$$

2. Total turn lengths: instead of the number of turns, this method uses the total length of turns uttered by the speaker.

$$
\mathcal{I}_{a, c}=\sum_{t \in\left[1, T_{c}\right]: a_{c, t}=a} N_{c, t}
$$


The two structural features used here capture the activeness of the speakers during a conversation and have been shown to be among the most effective features to detect influencers. These two structure-based methods are appropriate baselines in our experiment since, although being simple, they have been proven to be very effective in detecting influencers, both qualitatively (Bales 1970) and quantitatively (Rienks et al. 2006; Biran et al. 2012).

Topic-change-based methods score each instance based on features extracted from the posterior distributions of SITS.

1. Total topic shifts is the total number of expected topic shifts speaker $a$ makes in conversation $c$,

$$
\mathcal{I}_{a, c}=\sum_{t \in\left[1, T_{c}\right]: a_{c, t}=a} \bar{l}_{c, t}
$$

Recall that in SITS, each turn $t$ in conversation $c$ is associated with a binary latent variable $l_{c, t}$, which indicates whether the topic of turn $t$ is changed or not (these latent variables are introduced in Sect. 3). This expectation is computed through the empirical average of samples from the Gibbs sampler, $\bar{l}_{c, t}$, after a burn-in period. ${ }^{13}$ Intuitively, the higher $\bar{l}_{c, t}$ is, the more successful the speaker $a_{c, t}$ is in changing the topic of the conversation at this turn $t$.

2. Weighted topic shifts also quantify the topic changes a speaker makes by using the average topic shift indicator $\bar{l}_{c, t}$ but weighted by $\left(1-\pi_{a}\right)$, where $\pi_{a}$ is the topic shift tendency score of the speaker $a$. The basic idea here is that not all topic shifts should be counted equally. A successful topic shift by a speaker with small topic shift tendency score should be weighted higher than a successful topic by a speaker with high topic shift tendency score. The influence score of this ranking method is defined as

$$
\mathcal{I}_{a, c}=\left(1-\pi_{a}\right) \cdot \sum_{t \in\left[1, T_{c}\right]: a_{c, t}=a} \bar{l}_{c, t}
$$

\subsection{Experimental setup}

Datasets In this experiment, we use two datasets annotated for influencers: Crossfire and Wikipedia discussion pages. These two datasets and the annotation procedures are described in detail in Sect. 4. Table 8 shows dataset statistics.

Parameter settings and implementation As before, we use Gibbs sampling with 10 randomly initialized chains for inference. Initial hyperparameter values are sampled from $U(0,1)$ and statistics are collected after 200 burn-in iterations with a lag of 20 iterations over a total of 1000 iterations. Slice sampling optimizes the hyperparameters.

Evaluation measurements To evaluate the effectiveness of each ranking method in detecting the influencers, we use three standard evaluation measurements. The first measurement is $F_{1}$, the harmonic mean of precision and recall,

$$
F_{1}=\frac{2 \cdot \text { Precision } \cdot \text { Recall }}{\text { Precision }+ \text { Recall }}
$$

\footnotetext{
${ }^{13}$ For more details on how to compute this value, refer to Sect. 3 of Resnik and Hardisty (2010).
} 
Table 8 Statistics of the two datasets Crossfire and Wikipedia discussions that we annotated influencers. We use these two datasets to evaluate SITS on influencer detection

\begin{tabular}{lcr}
\hline Statistics & Crossfire & Wikipedia \\
\hline No. conversations & 3391 & 604 \\
No. unique speakers & 2381 & 1991 \\
Avg no. turns per conversation & 38.2 & 12.8 \\
Avg no. speakers per conversation & 5 & 7 \\
No. conversations annotated & 85 & 48 \\
No. positive instances & 197 & 57 \\
No. negative instances & 182 & 338 \\
\hline
\end{tabular}

Table 9 Influencer detection results on Crossfire and Wikipedia discussion pages. For both datasets, topicchange-based methods $(\star)$ outperform structure-based methods $(\diamond)$ by large margins. For all evaluation measurements, higher is better

\begin{tabular}{lllll}
\hline & Ranking methods & $F_{1}$ & AUC-ROC & AUC-PR \\
\hline Crossfire & Num. of turns $\diamond$ & .736 & .795 & .726 \\
& Total turn lengths $\diamond$ & .716 & .782 & .730 \\
& Total topic shifts $\star$ & $.806 \pm .0122$ & $.858 \pm .0068$ & $.865 \pm .0063$ \\
Wikipedia & Weighted topic shifts $\star$ & $.828 \pm .0100$ & $.869 \pm .0078$ & $.873 \pm .0057$ \\
& Num. of turns $\diamond$ & .367 & .730 & .291 \\
& Total turn lengths $\diamond$ & .306 & .732 & .281 \\
& Total topic shifts $\star$ & $.552 \pm .0353$ & $.752 \pm .0144$ & $.377 \pm .0284$ \\
& Weighted topic shifts $\star$ & $.488 \pm .0295$ & $.749 \pm .0149$ & $.379 \pm .0307$ \\
\hline
\end{tabular}

Even though $F_{1}$ is widely used, an important disadvantage is that it only examines a subset of top instances with highest scores, which might be the "easiest" cases. This phenomenon might lead to biased results when comparing the performance of different ranking methods. To overcome this problem, we also use AUC-ROC and AUC-PR, which measure the area under the Receiver-Operating-Characteristic (ROC) curve and the Precision-Recall (PR) curve. Using these two measurements, we can compare the performances of ranking methods using the full ranked lists. Davis and Goadrich (2006) point out that PR curve is more appropriate than ROC for skewed datasets.

\subsection{Results and analysis}

Table 9 shows the results of the four ranking methods using Crossfire and Wikipedia discussion datasets. Since we run our Gibbs samplers multiple times, the results of the two topic-change-based methods are reported with standard deviations (across different chains).

For both datasets, the two topic-change-based methods outperform the two structurebased methods by a large margin for all three evaluation measurements. The standard deviations in all three measurements of the two topic-change-based methods are relatively small. This shows the effectiveness of features based on topic changes in detecting influencers in 
conversations. In addition, the weighted topic shifts ranking method generally performs better than the total topic shifts method. This provides strong evidence that SITS is capable of capturing the speakers' propensity to change the topic. The improvement (if any) in the performance of the weighted topic shifts ranking method over the total topic shifts method is more obvious in the Crossfire dataset than in Wikipedia discussions. We argue that this is because conversations in Wikipedia discussion pages are generally shorter and contain more speakers than those in Crossfire debates. This leaves less evidence about the topic change behavior of the speakers in Wikipedia and thus SITS struggles to capture the speakers' behavior.

\section{Conclusions and future work}

SITS is a nonparametric hierarchical Bayesian model that jointly captures topics, topic shifts, and individuals' tendency to control the topic in conversations. SITS takes a nonparametric topic modeling approach, representing each turn in a conversation as a distribution over topics and consecutive turns' topic distributions as dependent on each other.

Crucially, SITS also models speaker-specific properties. As such, it improves performance on practical tasks such as unsupervised segmentation, but it also is attractive philosophically. Accurately modeling individuals is part of a broader research agenda that seeks to understand individuals' values (Fleischmann et al. 2011), interpersonal relationships (Chang et al. 2009a), and perspective (Hardisty et al. 2010), which creates a better understanding of what people think based on what they write or say (Pang and Lee 2008). One particularly interesting direction is to extend the model to capture how language is coordinated during the conversation and how it correlates with influence (Giles et al. 1991; Danescu-Niculescu-Mizil et al. 2012).

The problem of finding influencers in conversation has been studied for decades by researchers in communication, sociology, and psychology, who have long acknowledged qualitatively the correlation between the ability of a participant to control conversational topic and his or her influence on other participants during the conversation. With SITS, we now introduce a computational technique for modeling more formally who is controlling the conversation. Empirical results on the two datasets we annotated (Crossfire TV show and Wikipedia discussion pages) show that methods based on SITS outperform previous methods that used conversational structure patterns in detecting influencers.

Using an unsupervised statistical model for detecting influencers is an appealing choice because it extends easily to other languages and to corpora that are multilingual (Mimno et al. 2009; Boyd-Graber and Blei 2009). Moreover, topic models offer opportunities for exploring large corpora (Zhai et al. 2012) in a wide range of domains including political science (Grimmer 2009), music (Hoffman et al. 2009), programming source code (Andrzejewski et al. 2007) or even household archaeology (Mimno 2011). Recent work has created frameworks for interacting with statistical models (Hu et al. 2011) to improve the quality of the latent space (Chang et al. 2009b), understand relationships with other variables (Gardner et al. 2010), and allow the model to take advantage of expert knowledge (Andrzejewski et al. 2009) or knowledge resources (Boyd-Graber et al. 2007).

This work opens several future directions. First, even though associating each speaker with a scalar that models their tendency to change the topic does improve performance on both topic segmentation and influencer detection tasks, it is obviously an impoverished representation of an individual's conversational behaviors and could be enriched. For example, instead of just using a fixed parameter $\pi$ for each conversational participant, one could 
extend the model to capture evolving topic shift tendencies of participants during the conversation. Modeling individuals' perspective (Paul and Girju 2010), "side" (Thomas et al. 2006), or personal preferences for topics (Grimmer 2009) would also enrich the model and better illuminate the interaction of influence and topic.

Another important future direction is to extend the model to capture more explicitly the distinction between agenda setting and interaction influence. For example, questions or comments from the moderators during a political debate just shape the agenda of the debate and have little influence over how candidates would respond. Agenda setting does not have a direct effect on the views or opinions of others, and it does not try to sway the attitudes and beliefs of others. Agenda setting focuses generally on the topics that will be addressed, determining what those topics will be from the outset (McCombs and Reynolds 2009). It is during an interaction that an influencer is able to shape the discussion by shifting the interaction from one topic to another or providing evidence or expertise that can shape the opinions and judgments about the topics. To be identified as an influencer, however, others in the interaction must acknowledge or recognize the value of the expertise or agree with the opinion and viewpoints that have been offered. Thus, adding modules to find topic expertise (Marin et al. 2010) or agreement/disagreement (Galley et al. 2004) during the conversation would enable SITS to better detect influencers.

Understanding how individuals use language to influence others goes beyond conversational turn taking and topic control, however. In addition to what is said, often how something is expressed-i.e., the syntax —is nearly as important (Greene and Resnik 2009; Sayeed et al. 2012). Combining SITS with a model that can discover syntactic patterns (Sayeed et al. 2012) or multi-word expressions (Johnson 2010) associated with those attempting to influence a conversation would allow us to better understand how individuals use word choice and rhetorical strategies to persuade (Cialdini 2000; Anand et al. 2011) or coordinate with (Danescu-Niculescu-Mizil et al. 2012) others. Such systems could have a significant social impact, as they could identify, quantify, and measure attempts to spin or influence at a large scale. Models for automatic analysis of influence could lead to more transparent public conversations, ultimately improving our ability to achieve more considered and rational discussion of important topics, particularly in the political sphere.

Acknowledgements We would like to thank the reviewers for their insightful comments. We are grateful to Eric Hardisty, Pranav Anand, Craig Martell, Douglas W. Oard, Earl Wagner, and Marilyn Walker for helpful discussions. This research was funded in part by the Army Research Laboratory through ARL Cooperative Agreement W911NF-09-2-0072 and by the Office of the Director of National Intelligence (ODNI), Intelligence Advanced Research Projects Activity (IARPA), through the Army Research Laboratory. Jordan BoydGraber and Philip Resnik are supported by US National Science Foundation Grant NSF \#1018625. Viet-An Nguyen and Philip Resnik are also supported by US National Science Foundation Grant NSF \#IIS1211153. Any opinions, findings, conclusions, or recommendations expressed are the authors' and do not necessarily reflect those of the sponsors.

\section{Appendix A: Derivation of sampling equations}

\section{A.1 Nonparametric SITS}

In this section, we describe the general Gibbs sampler for our nonparametric model without using the minimal or maximal path assumption (Cowans 2006; Wallach 2008). The state space of our chain consists of the topic indices assigned to all tokens $z=\left\{z_{c, t, n}\right\}$ and topic shift indicators assigned to all turns $\boldsymbol{l}=\left\{l_{c, t}\right\}$. To obtain $z_{c, t, n}$ we need to know the path assigned for token $w_{c, t, n}$ through the hierarchy which includes table assignments $k_{c, t, n}^{\mathcal{T}}, k_{c, s, j}^{\mathcal{S}}$ 
Table 10 List of notations used

\section{Notation Descriptions}

$H \quad$ Base distribution over topics (a symmetric Dirichlet distribution)

$G_{0} \quad$ Distribution over topics drawn from $H$ and is shared across all conversations

$G_{c} \quad$ Distribution over topics drawn from $G_{0}$ for each conversation $c$ and is shared across all turns in $c$

$G_{c, t} \quad$ Distribution over topics drawn from $G_{c}$ for turn $t$ in conversation $c$

$G_{c, s} \quad$ Distribution over topics drawn from $G_{c}$ for segment $s$ in conversation $c$

$\phi_{k} \quad k$ th multinomial distribution over words (i.e. the $k$ th topic)

$\psi_{c, t, n} \quad$ Multinomial distribution over words generating token $w_{c, t, n}\left(\psi_{c, t, n} \equiv \phi_{z_{c, t, n}}\right)$

$l_{c, t} \quad$ Topic shift indicator assigned to turn $t$ of conversation $c$

$\pi_{m} \quad$ Topic shift tendency of speaker $m$

$w_{c, t, n} \quad n$th token of turn $t$ of conversation $c$

$a_{c, t} \quad$ Speaker of turn $t$ of conversation $c$

$s_{t} \quad$ Index of the segment that contains turn $t$

$N_{c, t} \quad$ Number of tokens in turn $t$ of conversations $c$

$T_{c, s} \quad$ Number of turns in segment $s$ of conversation $c$

$T_{c} \quad$ Number of turns in conversation $c$

$S_{c} \quad$ Number of segments in conversation $c$

$C \quad$ Number of conversations

$N_{c, s, j}^{\mathcal{S}} \quad$ Number of customers sitting at table $j$ in segment-level restaurant $c, s$

$N_{c, i} \mathcal{C} \quad$ Number of segment-level tables in restaurant $c$ assigned to conversation-level table $i$

$N_{k} \quad$ Number of conversation-level tables assigned to corpus-level table $k$

$k_{c, t, n}^{\mathcal{T}} \quad$ Index of table assigned to token $w_{c, t, n}$

$k_{c, s, j}^{\mathcal{S}} \quad$ Index of conversation-level table assigned to table $j$ in segment-level restaurant $c, s$

$k_{c, i}^{\mathcal{C}} \quad$ Index of corpus-level table assigned to table $i$ in conversation-level restaurant $c$

$J_{c, s}^{\mathcal{S}} \quad$ Number of tables in segment-level restaurant $c, s$

$I_{c}^{\mathcal{C}} \quad$ Number of tables in conversation-level restaurant $c$

$K^{+} \quad$ Number of tables in corpus-level restaurant

* The set of all hyperparameters

This notation is used to denote the marginal count

and $k_{c, i}^{\mathcal{C}}$. For ease of reference, the meaning of these symbols (and others used in this appendix) are listed in Table 10. Figure $7 \mathrm{~b}$ shows the relationship among the latent variables in our model. Once we know the three seating assignments $k_{c, t, n}^{\mathcal{T}}, k_{c, s, j}^{\mathcal{S}}$ and $k_{c, i}^{\mathcal{C}}, z_{c, t, n}$ can obtained by

$$
z_{c, t, n} \equiv k_{c, k_{c, s_{t}, k_{c, t, n}^{\mathcal{T}}}^{\mathcal{C}}}^{\mathcal{C}}
$$

To perform inference, we marginalize over all other latent variables and alternate between sampling paths $z$ and the sampling topic shift indicators $\boldsymbol{l}$.

\section{A.1.1 Sampling topic assignments}

Before deriving the sampling equations, we define $f_{k}^{-c, t, n}\left(w_{c, t, n}\right)$ to denote the conditional density of token $w_{c, t, n}$ under topic $k$ given all other items except $w_{c, t, n}$ 


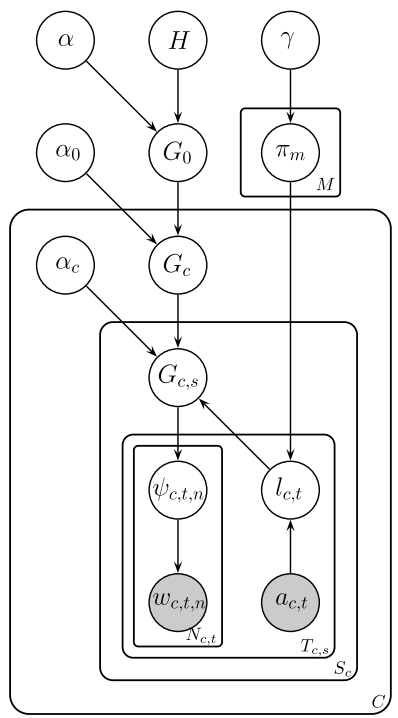

(a)

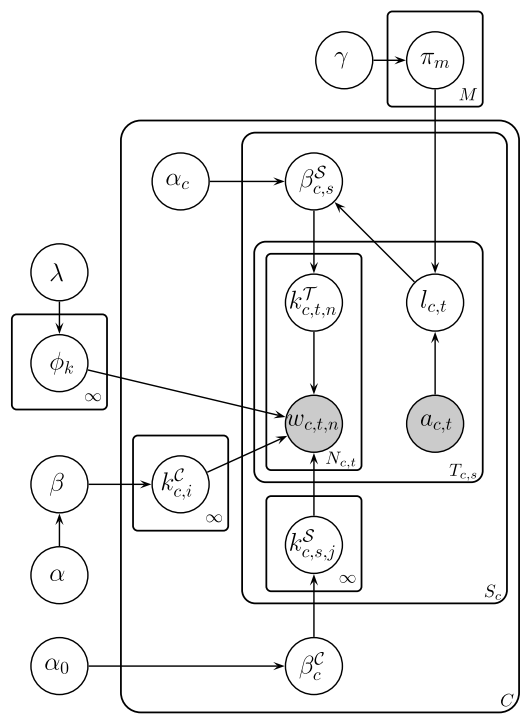

$\pi_{m} \sim \operatorname{Beta}(\gamma)$

$l_{c, t} \sim \operatorname{Bern}\left(\pi_{a_{c, t}}\right)$

$G_{0}=\sum_{k=1}^{\infty} \beta_{k} \delta_{\phi_{k}}$

$\beta \mid \alpha \sim \operatorname{GEM}(\alpha)$

$\phi_{k} \mid H(\lambda) \sim H(\lambda)$

$k_{c, i}^{\mathcal{C}} \mid \beta \sim \beta$

$G_{c}=\sum_{i=1}^{\infty} \beta_{c, i}^{\mathcal{C}} \delta_{\phi_{c, i}^{c}}$

$\beta_{c}^{\mathcal{C}} \mid \alpha_{0} \sim \operatorname{GEM}\left(\alpha_{0}\right)$

$\phi_{c, i}^{\mathcal{C}} \mid G_{0}\left(\lambda_{0}\right) \sim G_{0}\left(\lambda_{0}\right)$

$k_{c, s, j}^{\mathcal{S}} \mid \beta_{c}^{\mathcal{C}} \sim \beta_{c}^{\mathcal{C}}$

$G_{c, s}=\sum_{j=1}^{\infty} \beta_{c, s, j}^{\mathcal{S}} \delta_{\phi_{c, s, j}}^{\mathcal{S}}$

$\beta_{c, s}^{\mathcal{S}} \mid \alpha_{c}, \mathbf{l} \sim \operatorname{GEM}\left(\alpha_{c}\right)$

$\phi_{c, s, j}^{\mathcal{S}} \mid G_{c}\left(\lambda_{c}\right) \sim G_{c}\left(\lambda_{c}\right)$

$k_{c, t, n}^{\mathcal{T}} \mid \beta_{c, s(t)}^{\mathcal{S}} \sim \beta_{c, s(t)}^{\mathcal{S}}$

$z_{c, t, n}=k_{c, k_{c, s, k}^{s}, \tau_{c, n}^{\mathcal{T}}}^{\mathcal{T}}$

$w_{c, t, n} \sim \operatorname{Mult}\left(z_{c, t, n}\right)$

(b)

Fig. 7 Plate diagram representations of our nonparametric model: (a) representation using the notion of segment. We use $S_{c}$ to denote the number of segments in conversation $c$ and $T_{c, t}$ to denote the number of turns in segment $s$ of conversation $c ;(\mathbf{b})$ representation where explicit path assignments are shown

$$
f_{k}^{-c, t, n}\left(w_{c, t, n}\right)=\frac{\int_{\phi_{k}} P\left(\boldsymbol{w} \mid \phi_{k}\right) P\left(\phi_{k} \mid \lambda\right) \mathrm{d} \phi_{k}}{\int_{\phi_{k}} P\left(\boldsymbol{w}_{-c, t, n} \mid \phi_{k}\right) P\left(\phi_{k} \mid \lambda\right) \mathrm{d} \phi_{k}}= \begin{cases}\frac{M_{k, w_{c t n}+\lambda}}{M_{k, \cdot}+V \lambda}, & \text { if } k \text { exists; } \\ \frac{1}{V}, & \text { if } k \text { is new }\end{cases}
$$

where $M_{k, w}$ is the number of times word type $w$ assigned to topic $k, M_{k, \text {, represents marginal }}$ count, and superscript ${ }_{-c, t, n}$ is the same count excluding $w_{c, t, n}$.

To sample the path for a token (i.e. a customer), we take a similar approach to the first sampling method in Teh et al. (2006). We first sample a segment-level table $k_{c, t, n}^{\mathcal{T}}$ for customer $w_{c, t, n}$. The customer can sit at an existing table $j$ or create a new one, $j^{\text {new }}$. If a new segment-level table is created, a table in conversation-level restaurant $c$ will be sampled. Again, this $j^{\text {new }}$ can be assigned to an existing conversation-level table $i$ or a new one $i^{\text {new }}$. If $i^{\text {new }}$ is sampled, it can be assigned to an existing dish $k$ or a new one $k^{\text {new }}$ in the corpuslevel restaurant.

Sampling $k_{c, t, n}^{\mathcal{T}} \quad$ The probability of assigning a segment-level table $j$ to token $w_{c, t, n}$ is

$$
\begin{aligned}
& P\left(k_{c, t, n}^{\mathcal{T}}=j \mid \boldsymbol{k}_{-c, t, n}^{\mathcal{T}}, \boldsymbol{k}^{\mathcal{S}}, \boldsymbol{k}^{\mathcal{C}}, \boldsymbol{l}, \boldsymbol{w}, *\right) \\
& \quad \propto P\left(k_{c, t, n}^{\mathcal{T}}=j \mid \boldsymbol{k}_{-c, t, n}^{\mathcal{T}}\right) P\left(w_{c, t, n} \mid k_{c, t, n}^{\mathcal{T}}, \boldsymbol{k}_{-c, t, n}^{\mathcal{T}}, \boldsymbol{w}_{-c, t, n}, \boldsymbol{k}^{\mathcal{S}}, \boldsymbol{k}^{\mathcal{C}}, \boldsymbol{l}, *\right) \\
& \quad= \begin{cases}\frac{N_{c, s_{t}, j}^{\mathcal{S}}}{N_{c, s_{t},}^{\mathcal{S}}+\alpha_{c}} f_{k_{c, k_{c, s t}^{\mathcal{C}}, j}^{\mathcal{C}}, t, n}^{\mathcal{S}}\left(w_{c, t, n}\right), & \text { if } j \text { exists; } \\
\frac{\alpha_{c}}{N_{c, s_{t},}^{\mathcal{S}}+\alpha_{c}} P\left(w_{c, t, n} \mid k_{c, t, n}^{\mathcal{T}}=j^{n e w}, \boldsymbol{k}_{-c, t, n}^{\mathcal{T}}, \boldsymbol{w}_{-c, t, n}, \boldsymbol{k}^{\mathcal{S}}, \boldsymbol{k}^{\mathcal{C}}, \boldsymbol{l}, *\right), & \text { if } j \text { is new. }\end{cases}
\end{aligned}
$$


Marginalizing out all assignments of to $k_{c, s_{t}, j \text { new }}^{\mathcal{S}}$ (i.e. all possible tables $i$ 's including the new table $i^{\text {new }}$ of conversation-level restaurant $c$ ), we have:

$$
\begin{aligned}
& P\left(w_{c, t, n} \mid k_{c, t, n}^{\mathcal{T}}=j^{\text {new }}, \boldsymbol{k}_{-c, t, n}^{\mathcal{T}}, \boldsymbol{w}_{-c, t, n}, \boldsymbol{k}^{\mathcal{S}}, \boldsymbol{k}^{\mathcal{C}}, \boldsymbol{l}, *\right) \\
& =\sum_{i=1}^{I_{c}^{\mathcal{C}}} \frac{N_{c, i}^{\mathcal{C}}}{N_{c, \cdot}^{\mathcal{C}}+\alpha_{0}} f_{k_{c, i}^{\mathcal{C}}}^{-c, t, n}\left(w_{c, t, n}\right) \\
& \quad+\frac{\alpha_{0}}{N_{c, \cdot}^{\mathcal{C}}+\alpha_{0}} P\left(w_{c, t, n} \mid k_{c, s_{t}, j^{\text {new }}}^{\mathcal{S}}=i^{\text {new }}, \boldsymbol{k}_{-c, s_{t}, j^{n e w}}^{\mathcal{S}}, \boldsymbol{w}_{-c, t, n}, \boldsymbol{k}^{\mathcal{C}}, \boldsymbol{l}, *\right)
\end{aligned}
$$

Again, marginalizing out all possible assignments of $k_{c, i^{\text {new }}}^{\mathcal{C}}$ (i.e. all possible global dishes $k$ 's including a new $k^{\text {new }}$ ), we have:

$$
\begin{gathered}
P\left(w_{c, t, n} \mid k_{c, s_{t}, j^{n e w}}^{\mathcal{S}}=i^{\text {new }}, \boldsymbol{k}_{-c, s_{t}, j^{\text {new }}}^{\mathcal{S}}, \boldsymbol{w}_{-c, t, n}, \boldsymbol{k}^{\mathcal{C}}, \boldsymbol{l}, *\right) \\
\quad=\sum_{k=1}^{K} \frac{N_{k}}{N_{.}+\alpha} f_{k}^{-c, t, n}\left(w_{c, t, n}\right)+\frac{\alpha}{N_{.}+\alpha} f_{k^{\text {new }}}^{-c, t, n}\left(w_{c, t, n}\right)
\end{gathered}
$$

Sampling $k_{c, s, j}^{\mathcal{S}} \quad$ When a new segment-level table $j^{\text {new }}$ is created after sampling $k_{c, t, n}^{\mathcal{T}}$, we need to assign it to a table in the conversation-level restaurant. The probability of assigning it to conversation-level table $i$ is

$$
\begin{aligned}
& P\left(k_{c, s_{t}, j^{\text {new }}}^{\mathcal{S}}=i \mid \boldsymbol{k}_{-c, s_{t}, j^{\text {new }}}^{\mathcal{S}}, \boldsymbol{k}^{\mathcal{C}}, \boldsymbol{w}, \boldsymbol{l}, *\right) \\
& \quad \propto P\left(k_{c, s_{t}, j^{\text {new }}}^{\mathcal{S}}=i \mid \boldsymbol{k}_{-c, s_{t}, j^{\text {new }}}^{\mathcal{S}}\right) P\left(w_{c, t, n} \mid k_{c, s_{t}, j^{\text {new }}}^{\mathcal{S}}=i^{\text {new }}, \boldsymbol{k}_{-c, s_{t}, j^{\text {new }}}^{\mathcal{S}}, \boldsymbol{w}_{-c, t, n}, \boldsymbol{k}^{\mathcal{C}}, \boldsymbol{l}, *\right) \\
& \quad= \begin{cases}\frac{N_{c, i}^{\mathcal{C}}}{N_{c,}^{\mathcal{C}}+\alpha_{0}} f_{k_{c, i}^{\mathcal{C}, t, n}}^{-c,}\left(w_{c, t, n}\right), & \text { if } i \text { exists; } \\
\frac{\alpha_{0}}{N_{c,}^{\mathcal{C}}+\alpha_{0}} P\left(w_{c, t, n} \mid k_{c, s_{t}, j^{\text {new }}}^{\mathcal{S}}=i^{\text {new }}, \boldsymbol{k}_{-c, s_{t}, j^{\text {new }}}^{\mathcal{S}}, \boldsymbol{w}_{-c, t, n}, \boldsymbol{k}^{\mathcal{C}}, \boldsymbol{l}, *\right), & \text { if } i \text { is new. }\end{cases}
\end{aligned}
$$

The value of $P\left(w_{c, t, n} \mid k_{c, s_{t}, j^{\text {new }}}^{\mathcal{S}}=i^{\text {new }}, \boldsymbol{k}_{-c, s_{t}, j^{\text {new }}}^{\mathcal{S}}, \boldsymbol{w}_{-c, t, n}, \boldsymbol{k}^{\mathcal{C}}, \boldsymbol{l}, *\right)$ can be obtained in (18).

Sampling $k_{c, i}^{\mathcal{C}} \quad$ When a new conversation-level table $i^{\text {new }}$ is created, a table in the corpuslevel restaurant is sampled using the following probabilities

$$
P\left(k_{c, i^{\text {new }}}^{\mathcal{C}} \mid \boldsymbol{k}_{-c, i^{\text {new }}}^{\mathcal{C}}, \boldsymbol{w}, \boldsymbol{l}, *\right) \propto \begin{cases}\frac{N_{k}}{N \cdot+\alpha} f_{k}^{-c, t, n}\left(w_{c, t, n}\right), & \text { if } k \text { exists } \\ \frac{\alpha}{N .+\alpha} f_{k^{n e w}}^{-c, t, n}\left(w_{c, t, n}\right), & \text { if } k \text { is new. }\end{cases}
$$

By having all the seating assignments, we obtain the topic assignment $z_{c, t, n}$ for every token $w_{c, t, n}$ by using (14).

\section{A.1.2 Sampling topic shift indicators}

Given all the path assignments for all token $w_{c, t, n}$, we will sample the topic shift $l_{c, t}$ for every turn $t$ of conversation $c$. This probability is

$$
P\left(l_{c, t} \mid \boldsymbol{l}_{-c, t}, \boldsymbol{k}^{\mathcal{T}}, \boldsymbol{w}, \boldsymbol{a}, *\right) \propto P\left(l_{c, t} \mid \boldsymbol{l}_{-c, t}, \boldsymbol{a}, *\right) \cdot P\left(\boldsymbol{k}_{c, t}^{\mathcal{T}} \mid \boldsymbol{k}_{-c, t}^{\mathcal{T}}, l_{c, t}, \boldsymbol{l}_{-c, t}, *\right)
$$


Computing $P\left(l_{c, t} \mid \boldsymbol{l}_{-c, t}, \boldsymbol{a}, *\right) \quad$ The topic shifts $\boldsymbol{l}=\left\{l_{c, t}\right\}$ are drawn from a Bernoulli distribution parameterized by the topic shift tendency $\pi$, which is drawn from a conjugate prior $\operatorname{Beta}(\gamma)$. Marginalizing out $\pi$ we have

$$
P(\mathbf{l})=\int_{0}^{1} P(\mathbf{l} \mid \pi) P(\pi ; \gamma) \mathrm{d} \pi=\prod_{m=1}^{M} \frac{\Gamma(2 \gamma)}{\Gamma(\gamma)^{2}} \frac{\Gamma\left(S_{m, 1}+\gamma\right) \Gamma\left(S_{m, 0}+\gamma\right)}{\Gamma\left(S_{m, \cdot}+2 \gamma\right)}
$$

When $l_{c, t}=0$, the counts of number turns with topic shift indicator 1 will remain unchanged for all speakers. Similarly, for $l_{c, t}=1$. Thus, we have $P\left(l_{c, t} \mid \boldsymbol{l}_{-c, t}, \boldsymbol{a}, *\right)$ for two cases following Resnik and Hardisty (2010)

$$
P\left(l_{c, t} \mid \boldsymbol{l}_{-c, t}, \boldsymbol{a}, *\right)=\frac{P(\boldsymbol{l} \mid \boldsymbol{a}, *)}{P\left(\boldsymbol{l}_{-c, t} \mid \boldsymbol{a}, *\right)} \propto \begin{cases}\frac{s_{a_{c, t}, 0}^{-c, t}+\gamma}{S_{a_{c, t}, t}^{-c, t}, 2 \gamma}, & \text { if } l_{c, t}=0 \\ \frac{s_{a_{c, t}, 1}+\gamma}{s_{a_{c, t},+}^{-c, t}+2 \gamma}, & \text { if } l_{c, t}=1\end{cases}
$$

where $S_{a, x}^{-c, t}$ is the number of times speaker $a$ is assigned topic shift of value $x \in\{0,1\}$ excluding $l_{c, t}$.

Computing $P\left(\boldsymbol{k}_{c, t}^{\mathcal{T}} \mid \boldsymbol{k}_{-c, t}^{\mathcal{T}}, l_{c, t}, \boldsymbol{l}_{-c, t}, *\right)$

$$
P\left(\boldsymbol{k}_{c, t}^{\mathcal{T}} \mid \boldsymbol{k}_{-c, t}^{\mathcal{T}}, l_{c, t}, \boldsymbol{l}_{-c, t}, *\right) \propto \frac{P\left(\boldsymbol{k}_{c, t}^{\mathcal{T}}, \boldsymbol{k}_{-c, t}^{\mathcal{T}} \mid l_{c, t}, \boldsymbol{l}_{-c, t}, *\right)}{P\left(\boldsymbol{k}_{-c, t}^{\mathcal{T}} \mid l_{c, t}, \boldsymbol{l}_{-c, t}, *\right)}=\frac{P\left(\boldsymbol{k}^{\mathcal{T}} \mid l_{c, t}, \boldsymbol{l}_{-c, t}, *\right)}{P\left(\boldsymbol{k}_{-c, t}^{\mathcal{T}} \mid l_{c, t}, \boldsymbol{l}_{-c, t}, *\right)}
$$

Given all the customers assigned to all tables, the joint probability of all tables (Gershman and Blei 2012) is

$$
P\left(\boldsymbol{k}^{\mathcal{T}} \mid \boldsymbol{l}\right)=\prod_{c=1}^{C} \prod_{s=1}^{S_{c}} \frac{\alpha_{c}^{J_{c, s}^{\mathcal{S}}} \prod_{j=1}^{J_{c, s}^{\mathcal{S}}}\left(N_{c, s, j}^{\mathcal{S}}-1\right) !}{\prod_{x=1}^{N_{c, s, \cdot}^{\mathcal{S}}}\left(x-1+\alpha_{c}\right)}
$$

where $S_{c}$ is the number of segments in restaurant $c$. Substituting (25) into (24),

$$
\begin{aligned}
& P\left(\boldsymbol{k}_{c, t}^{\mathcal{T}} \mid l_{c, t}, \boldsymbol{l}_{-c, t}, \boldsymbol{k}_{-c, t}^{\mathcal{T}}\right)
\end{aligned}
$$

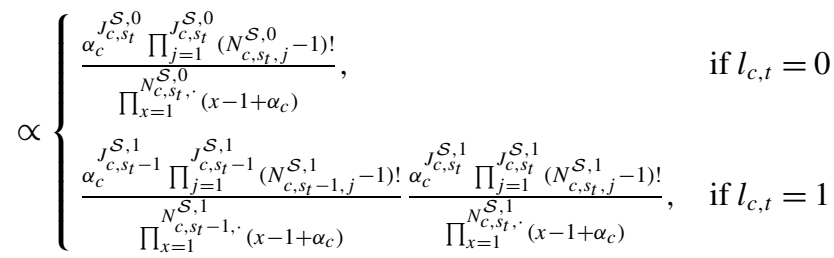

where

$-J_{c, s_{t}}^{\mathcal{S}, x}$ is the number of tables during segment $s$ of restaurant $c$ if $l_{c, t}=x$.

- $N_{c, s_{t}, j}^{\mathcal{S}, x}$ is the number of customers sitting at table $j$ during segment $s$ of restaurant $c$ if $l_{c, t}=x$. The marginal count $N_{c, s_{t}}^{\mathcal{S}, x}$, is the total number of customers during segment $s$ of restaurant $c$. 
Combining (23) and (26), we have the sampling equation for topic shift indicator from

$$
\begin{aligned}
& P\left(l_{c, t} \mid \boldsymbol{l}_{-c, t}, \boldsymbol{k}^{\mathcal{T}}, \boldsymbol{w}, \boldsymbol{a}, *\right)
\end{aligned}
$$

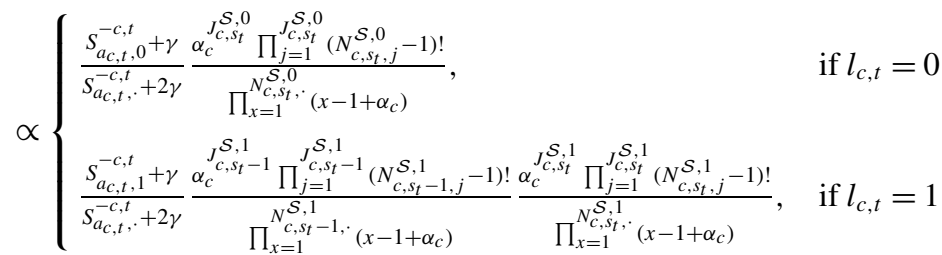

Thus, using (16), (19) and (20) to obtain the topic assignment $z_{c, t, n}$ (14) and using (27) to obtain the topic shift indicator $l_{c, t}$, we complete the derivation of sampling equations for our Gibbs samplers.

\section{A.2 Parametric SITS}

Like the nonparametric version, the state space of the Markov chain includes the topic indices assigned to all tokens $z=\left\{z_{c, t, n}\right\}$ and topic shifts assigned to all turns $\boldsymbol{l}=\left\{l_{c, t}\right\}$. Here, we present the sampling equations for both variables.

\section{A.2.3 Sampling topic assignments}

$$
\begin{aligned}
P & \left(z_{c, t, n}=y \mid z_{-c, t, n}, \boldsymbol{l}, \boldsymbol{w}, *\right) \\
& =\frac{P\left(z_{c, t, n}=y, z_{-c, t, n}, \boldsymbol{l}, \boldsymbol{w}\right)}{P\left(z_{-c, t, n}, \boldsymbol{l}, \boldsymbol{w}\right)} \\
& =\frac{P(\boldsymbol{w} \mid \boldsymbol{z}) P(\boldsymbol{z} \mid \boldsymbol{l}) P(\boldsymbol{l})}{P\left(\boldsymbol{w} \mid \boldsymbol{z}_{-c, t, n}\right) P\left(\boldsymbol{z}_{-c, t, n} \mid \boldsymbol{l}\right) P(\boldsymbol{l})}=\frac{P(\boldsymbol{w} \mid \boldsymbol{z})}{P\left(\boldsymbol{w} \mid \boldsymbol{z}_{-c, t, n}\right)} \frac{P(\boldsymbol{z} \mid \boldsymbol{l})}{P\left(\boldsymbol{z}_{-c, t, n} \mid \boldsymbol{l}\right)} \\
& \propto \frac{M_{y, w_{c, t, n}}^{-c, t, n}+\beta}{M_{y, \cdot, t, n}^{-c, n}+V \beta} \cdot \frac{N_{c, s_{t}, y}^{-c, n}+\alpha}{N_{c, s_{t}, n}^{-c, n}+K \alpha}
\end{aligned}
$$

where

- $M_{k, w}$ is the number of times that topic $k$ is assigned to token $w$ in the vocabulary.

- $N_{c, s, k}$ is the number of times topic $k$ is assigned to segment $s$ of conversation $c$.

- $V$ is the size of the vocabulary.

- $K$ is the number of predefined topics.

\section{A.2.4 Sampling topic shifts}

$$
\begin{aligned}
& P\left(l_{c, t} \mid \boldsymbol{l}_{-c, t}, \boldsymbol{z}, \boldsymbol{w}, \boldsymbol{a}, *\right) \\
& \propto \propto \begin{cases}\frac{S_{a_{c, t}, 0}^{-c, t}+\gamma}{S_{a_{c, t}, t}^{c, t}+2 \gamma} \cdot \frac{\prod_{k=1}^{K} \Gamma\left(N_{c, s_{t}, k}^{0}+\alpha\right)}{\Gamma\left(N_{c, s_{t},}^{0}+K \alpha\right)}, & \text { if } l_{c, t}=0 \\
\frac{S_{a_{c, t}, 1}^{-c, t}+\gamma}{S_{a_{c, t}, t}^{c, t}+2 \gamma} \cdot \frac{\Gamma(K \alpha)}{\Gamma(\alpha)^{K}} \frac{\prod_{k=1}^{K} \Gamma\left(N_{c, s_{t}-1, k}^{1}+\alpha\right)}{\Gamma\left(N_{c, s_{t}-1,+}^{1}+K \alpha\right)} \frac{\prod_{k=1}^{K} \Gamma\left(N_{c, s_{t}, k}^{1}+\alpha\right)}{\Gamma\left(N_{c, s_{t},}^{1}+K \alpha\right)}, & \text { if } l_{c, t}=1\end{cases}
\end{aligned}
$$

where 
- $S_{m, x}$ is the number of times that topic shift of value $x$ is assigned to speaker $m$.

- $N_{c, t, k}^{x}$ is the number of times that topic $k$ is assigned to segment $s$ of conversation $c$ if $l_{c, t}=x$.

\section{Appendix B: Crossfire annotation}

In this section we describe the procedures, definitions, and details of our process for annotating influencers.

Identifying influencers A discussant was considered an influencer if he or she initiated a topic shift that steered the conversation in a different direction, convinced others to agree to a certain viewpoint, or used an authoritative voice that caused others to defer to or reference that person's expertise. A discussant was not identified as an influencer if he or she merely initiated a topic at the start of a conversation, did not garner any support from others for the points he or she made, or was not recognized by others as speaking with authority.

Hosts The two hosts on Crossfire are tasked with changing the course of the discussion, as defined by their role on the program. Therefore, the hosts were considered annotated as influencers when they shifted the direction of the conversation. As a result, the hosts were generally included as influencers. To address this issue, up to four influencers could be identified within an interaction; then once all influencers were identified based on the interaction, the hosts were tagged within the influencers. In previous annotations, the number of influencers was limited to two per interaction. Allowing for up to four influencers and then tagging the hosts after all the influencers were identified allowed for guests to be tagged as influencers; otherwise they may have been left off because of the hosts' dominance.

Polite agreement Crossfire is a television show in which debate is the format and goal of the interaction, with the two hosts guiding the conversation. Certain patterns emerge from this type of debate, including some normative politeness that involves acknowledgement of another's point-such as polite agreement-but then making a shift in a way that counters that agreement. For example, when a discussant responded to another person by initially conceding a small point only to argue a larger point, that agreement was considered an argumentation tactic and not an expression of actual agreement; therefore, the initial agreement was not tagged as an indicator of an influencer.

- PRESS: Better marketing. Frank Gaffney, let's start by looking at those two polls back to back. The first one was a Gallup poll, which was December/January, released just about 10 days ago that surveyed residents of nine Muslim countries asking the question, do you think favorably about the U.S. or unfavorably, showed 22 percent only favorable opinion of the United States. 53 percent unfavorable. And then just a couple of days ago, CNN conducted its own poll here in the United States of Americans. Our opinion of Muslim nations, favorable opinion, 24 percent, unfavorable 41 percent. They're the mirror image of each other. So before you and I maybe tango about what we do about it, can we agree that this is a serious problem and we ought to be doing something about the spin war while we're fighting the military war?

- GAFFNEY: I think we can agree to that. It is a serious problem. I think the genesis of this problem, however, was not September 11. It certainly wasn't what we've been doing since September 11. I think it has its roots in fundamentally what most of the 
people of these countries have been receiving in the way of propaganda from governmentcontrolled media, al-Jazeera and similar kinds of outlets that frankly, given the vitriol against America that is pumped out by these media sources almost 24/7, it's surprising the poll numbers aren't worse for the United States in most of those countries.

Influencer NONE.

Reason GAFFNEY agrees on a small point in preparation to make a larger argument against what PRESS is claiming. GAFFNEY agrees that there is a problem with US-Arab relations, but disagrees with PRESS that the genesis of the problem was 9/11.

Video segments When discussants in the transcripts appeared in video clips, they were not considered as potential influencers; instead, video clips were treated as supporting material used, usually by the host, to make a point. Therefore, if a host showed a video that supported an argument and someone else agreed with the argument made in the video, then the influencer would be the host who introduced it.

- NOVAK: Congressman Menendez again, my new best friend, the former President Clinton, was asked on "LARRY KING" last night, all this stuff about 16 words, 10 words. He was asked whether biological and chemical weapons in Iraq when he was president, just a short time ago. Let's listen to what he said.

- CLINTON: When I left office there was a substantial amount of biological and chemical material unaccounted for.

- NOVAK: That was from "LARRY KING LIVE" last night. I mean, he is saying that there was material then. That's what the issue is. It isn't the issue of this parsing words and clipping off like Joe McCarthy does, the front side of the...

- PRYCE: ... that's exactly.

Influencer NOVAK.

Reason NOVAK is the influencer because he presents a recording of CLINTON to make his point, and PRYCE supports the argument in the video.

However, below is an example where the guest expressed agreement with what was said in the recording but disagreed with the argument the host was trying to make with the video. In this case, the guest provided a different interpretation of the video clip than the host had proposed.

- NOVAK: Senator Murray, welcome. When John Ashcroft, a former senator, was appointed-nominated by President- elect Bush, my Senate sources said if the vote were taken right then, there would be more than 10 votes against him. How did it get up to 42 votes against him? I think the code was broken today by the Senate-the chairman of the Senate Judiciary Committee. And let's listen to Orrin Hatch and how he explains it.

- SEN. ORRIN HATCH (R-UT), CHAIRMAN, JUDICIARY COMMITTEE: This is one of the worst episodes in the onward trashing soldiers routine that I've ever seen. Outside groups basically cowing United States senators, telling them that they all have primaries and that they'll do everything they can to defeat them unless they send this message to President Bush.

- NOVAK: Isn't that right? You and your companions buckled under to the vast left-wing conspiracy. $* * *$

- DREIER: I don't think it means that all, Bill. I mean, if you look at what has taken place here, it's very obvious to me that, as Orrin Hatch said, there are a wide range of groups that led the American people to say the things that they did to my colleague Patty Murray, to me, and a wide range of others. And I just happen to believe that it's a mischaracterization 
of the record of John Ashcroft. John Ashcroft is someone who, as we all know, was the chairman of the National Governor's Association. He was the chairman of the Attorney General's Association, selected by his peers to that position. They tried to paint him as some kind of extremist, and I don't believe that George Bush will be appointing nominees to the United States Supreme Court or to any other spots who are deserving of the kind of criticism that was leveled at John Ashcroft. So, it's true, Bill, that these groups have gotten out there and successfully convinced many that John Ashcroft is something other than what he is. And the statement that Bob just put up on to the screen, that was made during the hearings and I listened to the hearings, I mean, it's very clear that John Ashcroft offered very thoughtful answers to the questions and I believe was very deserving of broader support than he got.

Influencer NOVAK.

Reason NOVAK is the influencer because he presents a recording of SEN. ORRIN HATCH, and DREIER supports the argument in the video about why Ashcroft faced great criticism.

Shift in topic At times, guests were able to shift the topic effectively, but not for too long because the hosts would jump in and shift the topic back or in a different direction. Nonetheless, this effort to shift the topic was viewed as a topic shift, as in the following example.

- NOVAK: Well, senator, you have been a very successful electoral politician, and you've never found the necessity to move to the center. But don't you think very possibly that Al Gore, assuming he's going to be nominated, is going to have to get away from this extremist politics, and try to get—at least pose as a centrist? Don't you feel that...

- BOXER: Bobby, Bob, thank you for saying I'm a successful politician. But I have to say to you this. As much as you would like to make these campaigns about left and right, and actually you do that on this show all the time, so I hate to undercut you...

- NOVAK: Yes, we do.

- BOXER: ... it's really about what people want, Bob. It's about opportunity, education, a good economy, a decent minimum wage. When George Bush gets up and defends - and I'd like to see this, both Paul and I-a \$3.35 minimum wage, I want to see what people think. Is this compassionate? So these are the issues we have to deal with.

- NOVAK: Well, let me-let me-let me try-let me try...

- BOXER: It's not...

- WELLSTONE: Do you think, Bob, in a cafe in Minnesota, anybody ever comes up to me and says, Paul, are you left, are you right, or are you center?

- NOVAK: Well, they know where you are, they know where you are, Paul.

- WELLSTONE: They-no, no, no, no, no. They talk very concrete issues, about jobs, about their children and education...

- NOVAK: Minnesota's a funny place.

- WELLSTONE: ... about health care. So do people in the country.

- NOVAK: Senator Boxer, let me try something else.

- BOXER: Try it again, Bob.

- WELLSTONE: Yes, try it, we're ready, we're ready. Go ahead and try it, we're ready.

\section{Influencer BOXER.}

Reason BOXER is an influencer because she changes the topic and challenges the premise of NOVAK's question about right versus left, and WELLSTONE joins her in challenging NOVAK on this point. 


\section{References}

Abbott, R., Walker, M., Anand, P., Fox Tree, J. E., Bowmani, R., \& King, J. (2011). How can you say such things?!?: Recognizing disagreement in informal political argument. In Proceedings of the workshop on language in social media (LSM).

Ahmed, A., \& Xing, E. P. (2008). Dynamic non-parametric mixture models and the recurrent Chinese restaurant process: with applications to evolutionary clustering. In Proceedings of SIAM international conference on data mining.

Ahmed, A., \& Xing, E. P. (2010). Timeline: a dynamic hierarchical Dirichlet process model for recovering birth/death and evolution of topics in text stream. In Proceedings of uncertainty in artificial intelligence.

Alarcon-del Amo, M., Lorenzo-Romero, C., \& Gomez-Borja, M. (2011). Classifying and profiling social networking site users: a latent segmentation approach. Cyberpsychology, Behavior, and Social Networking, 14(9).

Anand, P., King, J., Boyd-Graber, J., Wagner, E., Martell, C., Oard, D. W., \& Resnik, P. (2011). Believe me: we can do this! In The AAAI 2011 workshop on computational models of natural argument.

Andrzejewski, D., Mulhern, A., Liblit, B., \& Zhu, X. (2007). Statistical debugging using latent topic models. In Proceedings of European conference of machine learning.

Andrzejewski, D., Zhu, X., \& Craven, M. (2009). Incorporating domain knowledge into topic modeling via Dirichlet forest priors. In Proceedings of the international conference of machine learning.

Antoniak, C. E. (1974). Mixtures of Dirichlet processes with applications to Bayesian nonparametric problems. The Annals of Statistics, 2(6), 1152-1174.

Aran, O., \& Gatica-Perez, D. (2010). Fusing audio-visual nonverbal cues to detect dominant people in group conversations. In Proceedings of the international conference on pattern recognition (ICPR).

Artstein, R., \& Poesio, M. (2008). Inter-coder agreement for computational linguistics. Computational Linguistics, 34(4), 555-596.

Bales, R. F. (1970). Personality and interpersonal behavior. New York: Holt, Rinehart, and Winston.

Basu, S., Choudhury, T., Clarkson, B., \& Pentland, A. S. (2001). Learning human interactions with the influence model (Tech. Rep. 539). MIT Media Laboratory.

Beeferman, D., Berger, A., \& Lafferty, J. (1999). Statistical models for text segmentation. Machine Learning, 34(1-3), 177-210.

Bender, E. M., Morgan, J. T., Oxley, M., Zachry, M., Hutchinson, B., Marin, A., Zhang, B., \& Ostendorf, M. (2011). Annotating social acts: authority claims and alignment moves in wikipedia talk pages. In Proceedings of the workshop on languages in social media (LSM).

Biran, O., Rosenthal, S., Andreas, J., McKeown, K., \& Rambow, O. (2012). Detecting influencers in written online conversations. In Proceedings of the workshop on language in social media (LSM).

Blau, P. (1964). Exchange and power in social life. Sociology political science. Transaction Books.

Blei, D. M. (2012). Probabilistic topic models. Communications of the ACM, 55(4), 77-84.

Blei, D. M., \& Lafferty, J. D. (2006). Dynamic topic models. In Proceedings of the international conference of machine learning.

Blei, D. M., Ng, A. Y., \& Jordan, M. I. (2003). Latent Dirichlet allocation. Journal of Machine Learning Research, 3, 993-1022.

Booth, N., \& Matic, A. (2011). Mapping and leveraging influencers in social media to shape corporate brand perceptions. Corporate Communications, 16(3), 184-191.

Boyd-Graber, J., \& Blei, D. M. (2008). Syntactic topic models. In Proceedings of advances in neural information processing systems.

Boyd-Graber, J., \& Blei, D. M. (2009). Multilingual topic models for unaligned text. In Proceedings of uncertainty in artificial intelligence.

Boyd-Graber, J., Blei, D. M., \& Zhu, X. (2007). A topic model for word sense disambiguation. In Proceedings of empirical methods in natural language processing.

Boydstun, A. E., Glazier, R. A., \& Phillips, C. (2013). Agenda control in the 2008 presidential debates. American Politics Research.

Brooke, M. E., \& Ng, S. H. (1986). Language and social influence in small conversational groups. Journal of Language and Social Psychology, 5(3), 201-210.

Burrel, N. A., \& Koper, R. J. (1998). The efficacy of powerful/powerless language on attitudes and source credibility. In Persuasion: advances through meta-analysis.

Butler, B., Joyce, E., \& Pike, J. (2008). Don't look now, but we've created a bureaucracy: the nature and roles of policies and rules in wikipedia. In International conference on human factors in computing systems.

Chang, J., Boyd-Graber, J., \& Blei, D. M. (2009a). Connections between the lines: augmenting social networks with text. In Knowledge discovery and data mining.

Chang, J., Boyd-Graber, J., Wang, C., Gerrish, S., \& Blei, D. M. (2009b). Reading tea leaves: how humans interpret topic models. In Proceedings of advances in neural information processing systems. 
Chen, H., Branavan, S. R. K., Barzilay, R., \& Karger, D. R. (2009). Global models of document structure using latent permutations. In Computational linguistics.

Choi, F. Y. Y., Wiemer-Hastings, P., \& Moore, J. (2001). Latent semantic analysis for text segmentation. In Proceedings of empirical methods in natural language processing.

Cialdini, R. B. (2000). Influence: science and practice (4th ed.). Needham Heights: Allyn \& Bacon.

Cortes, C., \& Vapnik, V. (1995). Support-vector networks. Machine Learning, 20(3), 273-297.

Cowans, P. J. (2006). Probabilistic document modelling. Ph.D. thesis, University of Cambridge.

Daley, J. A., McCroskey, J. C., \& Richmond, V. P. (1977). Relationships between vocal activity and perception of communicators in small group interaction. Western Journal of Speech Communication, 41(3), 175-187.

Danescu-Niculescu-Mizil, C., Lee, L., Pang, B., \& Kleinberg, J. (2012). Echoes of power: language effects and power differences in social interaction. In Proceedings of world wide web conference (pp. 699708).

Davis, J., \& Goadrich, M. (2006). The relationship between precision-recall and ROC curves. In Proceedings of the international conference of machine learning.

Dowman, M., Savova, V., Griffiths, T. L., Kording, K. P., Tenenbaum, J. B., \& Purver, M. (2008). A probabilistic model of meetings that combines words and discourse features. Transactions on Audio, Speech, and Language Processing, 16(7), 1238-1248.

Drake, B. H., \& Moberg, D. J. (1986). Communicating influence attempts in dyads: linguistic sedatives and palliatives. The Academy of Management Review, 11(3), 567-584.

Du, L., Buntine, W. L., \& Jin, H. (2010). Sequential latent Dirichlet allocation: discover underlying topic structures within a document. In International conference on data mining.

Ehlen, P., Purver, M., \& Niekrasz, J. (2007). A meeting browser that learns. In Proceedings of the AAAI spring symposium on interaction challenges for intelligent assistants.

Eisenstein, J., \& Barzilay, R. (2008). Bayesian unsupervised topic segmentation. In Proceedings of empirical methods in natural language processing.

Emerson, R. M. (1981). Social exchange theory. In M. Rosenberg \& R. H. Turner (Eds.), Social psychology: sociological perspectives (pp. 30-65). New York: Basic Books.

Fallows, J. (2008). Your VP debate wrapup in four bullet points. The Atlantic. http://www.theatlantic.com/ technology/archive/2008/10/your-vp-debate-wrapup-in-four-bullet-points/8887/.

Ferguson, T. S. (1973). A Bayesian analysis of some nonparametric problems. The Annals of Statistics, 1(2), 209-230.

Fleischmann, K. R., Templeton, T. C., \& Boyd-Graber, J. (2011). Modeling diverse standpoints in text classification: learning to be human by modeling human values. In iConference.

Foa, U. G., \& Foa, E. B. (1972). Resource exchange: toward a structural theory of interpersonal communication. In A. W. Siegman \& B. Pope (Eds.), Studies in dyadic communication (pp. 291-325). Elmsford: Pergamon.

Fox, E. B., Sudderth, E. B., Jordan, M. I., \& Willsky, A. S. (2008). An HDP-HMM for systems with state persistence. In Proceedings of the international conference of machine learning.

Galley, M., McKeown, K., Fosler-Lussier, E., \& Jing, H. (2003). Discourse segmentation of multi-party conversation. In Proceedings of the association for computational linguistics.

Galley, M., McKeown, K., Hirschberg, J., \& Shriberg, E. (2004). Identifying agreement and disagreement in conversational speech: use of Bayesian networks to model pragmatic dependencies. In Proceedings of the association for computational linguistics.

Gardner, M., Lutes, J., Lund, J., Hansen, J., Walker, D., Ringger, E., \& Seppi, K. (2010). The topic browser: an interactive tool for browsing topic models. In Proceedings of advances in neural information processing systems.

Gershman, S. J., \& Blei, D. M. (2012). A tutorial on Bayesian nonparametric models. Journal of Mathematical Psychology, 56(1), 1-12.

Giles, H., Coupland, N., \& Coupland, J. (1991). Accommodation theory: communication, context, and consequence. In H. Giles, N. Coupland, \& J. Coupland (Eds.), Contexts of accommodation: developments in applied socio-linguistics (pp. 1-68). Cambridge: Cambridge University Press.

Greene, S., \& Resnik, P. (2009). More than words: syntactic packaging and implicit sentiment. In NAACL.

Grimmer, J. (2009). A Bayesian hierarchical topic model for political texts: measuring expressed agendas in senate press releases. Political Analysis, 18(1), 1-35.

Halliday, M., \& Hasan, R. (1976). Cohesion in English. New York: Longman.

Hamilton, M. A., \& Hunter, J. E. (1998). The effect of language intensity on receiver evaluations of message, source, and topic. In Persuasion: advances through meta-analysis.

Hardisty, E., Boyd-Graber, J., \& Resnik, P. (2010). Modeling perspective using adaptor grammars. In Proceedings of empirical methods in natural language processing. 
Hawes, T., Lin, J., \& Resnik, P. (2009). Elements of a computational model for multi-party discourse: the turn-taking behavior of supreme court justices. Journal of the American Society for Information Science and Technology, 60(8), 1607-1615.

Hearst, M. A. (1997). TextTiling: Segmenting text into multi-paragraph subtopic passages. Computational Linguistics, 23(1), 33-64.

Hirschberg, J., \& Litman, D. (1993). Empirical studies on the disambiguation of cue phrases. Computational Linguistics, 19(3), 501-530.

Hoffman, M. D., Blei, D. M., \& Cook, P. R. (2009). Finding latent sources in recorded music with a shiftinvariant hdp. In Proceedings of the conference on digital audio effects.

Horton, S. (2008). The Ifill factor. Harpers Magazine. http://harpers.org/archive/2008/10/hbc-90003659.

Hsueh, P., Moore, J. D., \& Renals, S. (2006). Automatic segmentation of multiparty dialogue. In Proceedings of the European chapter of the association for computational linguistics.

$\mathrm{Hu}$, Y., Boyd-Graber, J., \& Satinoff, B. (2011). Interactive topic modeling. In Proceedings of the association for computational linguistics.

Huffaker, D. (2010). Dimensions of leadership and social influence in online communities. Human Communication Research, 36(4), 593-617.

Hung, H., Huang, Y., Friedland, G., \& Gatica-Perez, D. (2011). Estimating dominance in multi-party meetings using speaker diarization. Transactions on Audio, Speech, and Language Processing, 19(4), 847-860.

Ireland, M. E., Slatcher, R. B., Eastwick, P. W., Scissors, L. E., Finkel, E. J., \& Pennebaker, J. W. (2011). Language style matching predicts relationship initiation and stability. Psychological Science, 22(1), 3944.

Janin, A., Baron, D., Edwards, J., Ellis, D., Gelbart, D., Morgan, N., Peskin, B., Pfau, T., Shriberg, E., Stolcke, A., \& Wooters, C. (2003). The ICSI meeting corpus. In IEEE international conference on acoustics, speech, and signal processing.

Jayagopi, D. B., Hung, H., Yeo, C., \& Gatica-Perez, D. (2009). Modeling dominance in group conversations using nonverbal activity cues. Transactions on Audio, Speech, and Language Processing, 17(3), 501513.

Johnson, M. (2010). PCFGs, topic models, adaptor grammars and learning topical collocations and the structure of proper names. In Proceedings of the association for computational linguistics.

Katz, E., \& Lazarsfeld, P. F. (1955). Personal influence: the part played by people in the flow of mass communications. Foundations of communications research. New York: Free Press.

Kellermann, K. (2004). Topical profiling: emergent, co-occurring, and relationally defining topics in talk. Journal of Language and Social Psychology, 23(3), 308-337.

Marin, A., Ostendorf, M., Zhang, B., Morgan, J. T., Oxley, M., Zachry, M., \& Bender, E. M. (2010). Detecting authority bids in online discussions. In $S L T$ (pp. 49-54).

Mast, M. S. (2002). Dominance as expressed and inferred through speaking time. Human Communication Research, 28(3), 420-450.

McCombs, M., \& Reynolds, A. (2009). How the news shapes our civic agenda. In J. Bryant \& M. B. Oliver (Eds.), Media effects: advances in theory and research (pp. 1-16). Lawrence Erlbaum.

Mimno, D. M. (2011). Reconstructing Pompeian households. In Proceedings of uncertainty in artificial intelligence (pp. 506-513).

Mimno, D., Wallach, H., Naradowsky, J., Smith, D., \& McCallum, A. (2009). Polylingual topic models. In Proceedings of empirical methods in natural language processing.

Morris, J., \& Hirst, G. (1991). Lexical cohesion computed by thesaural relations as an indicator of the structure of text. Computational Linguistics, 17(1), 21-48.

Müller, P., \& Quintana, F. A. (2004). Nonparametric Bayesian data analysis. Statistical Science, 19(1), 95110.

Murray, G., Renals, S., \& Carletta, J. (2005). Extractive summarization of meeting recordings. In European conference on speech communication and technology.

Neal, R. M. (2000). Markov chain sampling methods for Dirichlet process mixture models. Journal of Computational and Graphical Statistics, 9(2), 249-265.

Neal, R. M. (2003). Slice sampling. The Annals of Statistics, 31, 705-767.

Ng, S. H., \& Bradac, J. J. (1993). Power in language: verbal communication and social influence. language and language behaviors. Thousand Oaks: Sage Publications.

Ng, S. H., Bell, D., \& Brooke, M. (1993). Gaining turns and achieving high influence ranking in small conversational groups. British Journal of Social Psychology, 32(3), 265-275.

Nguyen, V. A., Boyd-Graber, J., \& Resnik, P. (2012). SITS: a hierarchical nonparametric model using speaker identity for topic segmentation in multiparty conversations. In Proceedings of the association for computational linguistics.

Olney, A., \& Cai, Z. (2005). An orthonormal basis for topic segmentation in tutorial dialogue. In Proceedings of the human language technology conference. 
Otsuka, K., Yamato, J., Takemae, Y., \& Murase, H. (2006). Quantifying interpersonal influence in face-toface conversations based on visual attention patterns. In International conference on human factors in computing systems.

Palmer, M. T. (1989). Controlling conversations: turns, topics and interpersonal control. Communication Monographs, 56(1), 1-18.

Pang, B., \& Lee, L. (2008). Opinion mining and sentiment analysis. Hanover: Now Publishers.

Passonneau, R. J., \& Litman, D. J. (1997). Discourse segmentation by human and automated means. Computational Linguistics, 23(1), 103-139.

Paul, R. (2007). Political power and the rule of law. Texas Straight Talk. http://www.lewrockwell.com/paul/ paul366.html.

Paul, M., \& Girju, R. (2010). A two-dimensional topic-aspect model for discovering multi-faceted topics. In Association for the advancement of artificial intelligence.

Pele, O., \& Werman, M. (2008). A linear time histogram metric for improved sift matching. In ECCV (pp. 495-508).

Pele, O., \& Werman, M. (2009). Fast and robust earth mover's distances. In International conference on computer vision.

Pevzner, L., \& Hearst, M. A. (2002). A critique and improvement of an evaluation metric for text segmentation. Computational Linguistics, 28(1), 19-36.

Planalp, S., \& Tracy, K. (1980). Not to change the topic but ...: a cognitive approach to the management of conversations. In Communication yearbook 4, New Brunswick (pp. 237-258).

Purver, M. (2011). Topic segmentation. In Spoken language understanding: systems for extracting semantic information from speech.

Purver, M., Körding, K., Griffiths, T. L., \& Tenenbaum, J. (2006). Unsupervised topic modelling for multiparty spoken discourse. In Proceedings of the association for computational linguistics.

Regula, R., \& Julian, W. (1973). The impact of quality and frequency of task contributions on perceived ability. The Journal of Social Psychology, 89(1), 115-122.

Reid, S. A., \& Ng, S. H. (2000). Conversation as a resource for influence: evidence for prototypical arguments and social identification processes. European Journal of Social Psychology, 30(1), 83-100.

Ren, L., Dunson, D. B., \& Carin, L. (2008). The dynamic hierarchical Dirichlet process. In Proceedings of the international conference of machine learning.

Resnik, P., \& Hardisty, E. (2010). Gibbs sampling for the uninitiated (Tech. Rep. UMIACS-TR-2010-04). University of Maryland. http://drum.lib.umd.edu//handle/1903/10058.

Rienks, R., \& Heylen, D. (2005). Dominance detection in meetings using easily obtainable features. In Proceedings of the 2 nd joint workshop on multimodal interaction and related machine learning algorithms.

Rienks, R., Zhang, D., Gatica-Perez, D., \& Post, W. (2006). Detection and application of influence rankings in small group meetings. In Proceedings of the international conference on multimodal interfaces, ICMI '06.

Rubner, Y., Tomasi, C., \& Guibas, L. J. (2000). The earth mover's distance as a metric for image retrieval. International Journal of Computer Vision, 40(2), 99-121.

Sayeed, A. B., Boyd-Graber, J., Rusk, B., \& Weinberg, A. (2012). Grammatical structures for word-level sentiment detection. In North American association of computational linguistics.

Scheer, L. K., \& Stern, L. W. (1992). The effect of influence type and performance outcomes on attitude toward the influencer. Journal of Marketing Research, 29(1), 128-142.

Schlenker, B. R., Nacci, P., Helm, B., \& Tedeschi, J. T. (1976). Reactions to coercive and reward power: the effects of switching influence modes on target compliance. Sociometry, 39(4), 316-323.

Sorrentino, R. M., \& Boutiller, R. G. (1972). The effect of quantity and quality of verbal interaction on ratings of leadership ability. Journal of Experimental Social Psychology, 5, 403-411.

Stang, D. J. (1973). Effect of interaction rate on ratings of leadership and liking. Journal of Personality and Social Psychology, 27(3), 405-408.

Teh, Y. W. (2006). A hierarchical Bayesian language model based on Pitman-Yor processes. In Proceedings of the association for computational linguistics.

Teh, Y. W., Jordan, M. I., Beal, M. J., \& Blei, D. M. (2006). Hierarchical Dirichlet processes. Journal of the American Statistical Association, 101(476), 1566-1581.

Thomas, M., Pang, B., \& Lee, L. (2006). Get out the vote: determining support or opposition from congressional floor-debate transcripts. In Proceedings of empirical methods in natural language processing.

Trammell, K. D., \& Keshelashvili, A. (2005). Examining the new influencers: a self-presentation study of a-list blogs. Journalism \& Mass Communication Quarterly, 82(4), 968-982.

Tur, G., Stolcke, A., Voss, L., Peters, S., Hakkani-Tür, D., Dowding, J., Favre, B., Fernández, R., Frampton, M., Frandsen, M., Frederickson, C., Graciarena, M., Kintzing, D., Leveque, K., Mason, S., Niekrasz, J., Purver, M., Riedhammer, K., Shriberg, E., Tien, J., Vergyri, D., \& Yang, F. (2010). The CALO meeting assistant system. Transactions on Audio, Speech, and Language Processing, 18(6), 1601-1611. 
Wallach, H. M. (2006). Topic modeling: beyond bag-of-words. In Proceedings of the international conference of machine learning.

Wallach, H. M. (2008). Structured topic models for language. Ph.D. thesis, University of Cambridge.

Wang, C., Blei, D. M., \& Heckerman, D. (2008). Continuous time dynamic topic models. In Proceedings of uncertainty in artificial intelligence.

Weimann, G. (1994). The influentials: people who influence people. Suny series in human communication processes. Albany: State University of New York Press.

Zhai, K., Boyd-Graber, J., Asadi, N., \& Alkhouja, M. (2012). Mr. LDA: a flexible large scale topic modeling package using variational inference in mapreduce. In Proceedings of world wide web conference.

Zhang, D., Gatica-Perez, D., Bengio, S., \& Roy, D. (2005). Learning influence among interacting Markov chains. In Proceedings of advances in neural information processing systems. 